\title{
Metabolomics Profiles of Smokers from Two Ethnic Groups with Differing Lung Cancer Risk
}

Romel Dator ${ }^{1}$, Peter W. Villalta ${ }^{1}$, Nicole Thomson ${ }^{1}$, Joni Jensen ${ }^{4}$, Dorothy K. Hatsukami ${ }^{1}{ }^{4}$, Irina Stepanov ${ }^{1,3}$, Benedikt Warth ${ }^{2,5^{*}}$, and Silvia Balbo ${ }^{1,3^{*}}$

${ }^{1}$ Masonic Cancer Center, University of Minnesota, Minneapolis, MN 55455, United States

${ }^{2}$ Department of Food Chemistry and Toxicology, Faculty of Chemistry, University of Vienna, Währingerstraße 38, 1090 Vienna, Austria

${ }^{3}$ Division of Environmental Health Sciences, School of Public Health, University of Minnesota, Minneapolis, MN 55455, United States

${ }^{4}$ Department of Psychiatry, University of Minnesota, Minneapolis, MN 55455, United States ${ }^{5}$ Scripps Center for Metabolomics, The Scripps Research Institute, La Jolla, CA 92037, United States

*Senior corresponding authors

Phone: (612) 624-4240

Fax: $\quad(612) 624-3869$

Email: balbo006@umn.edu; benedikt.warth@univie.ac.at 
Figure $\mathbf{S 1}$

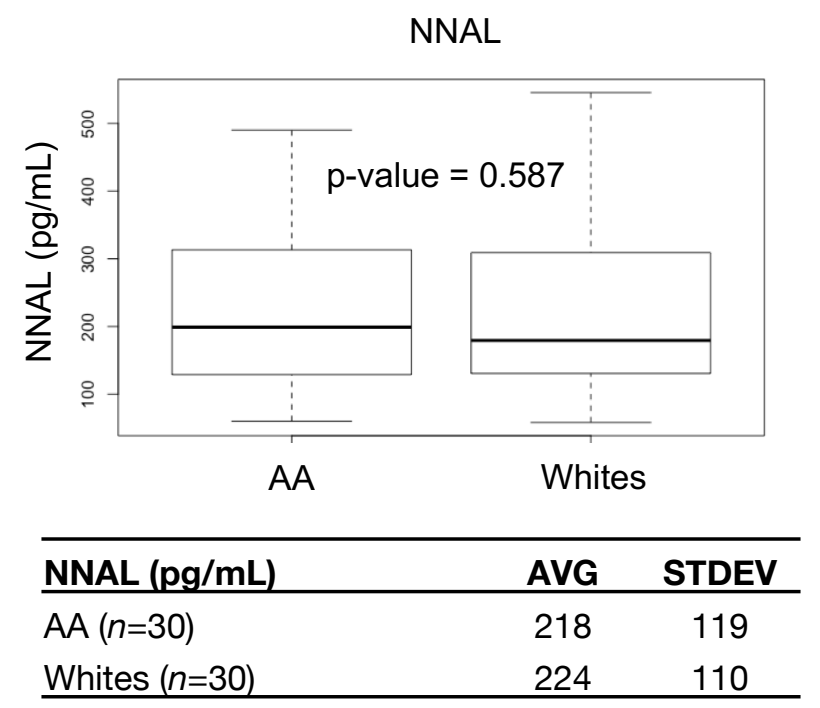

Fig. S1. Levels of NNAL $(\mathrm{pg} / \mathrm{mL})$ in urine of the 60 subjects used in the metabolomics analysis. 

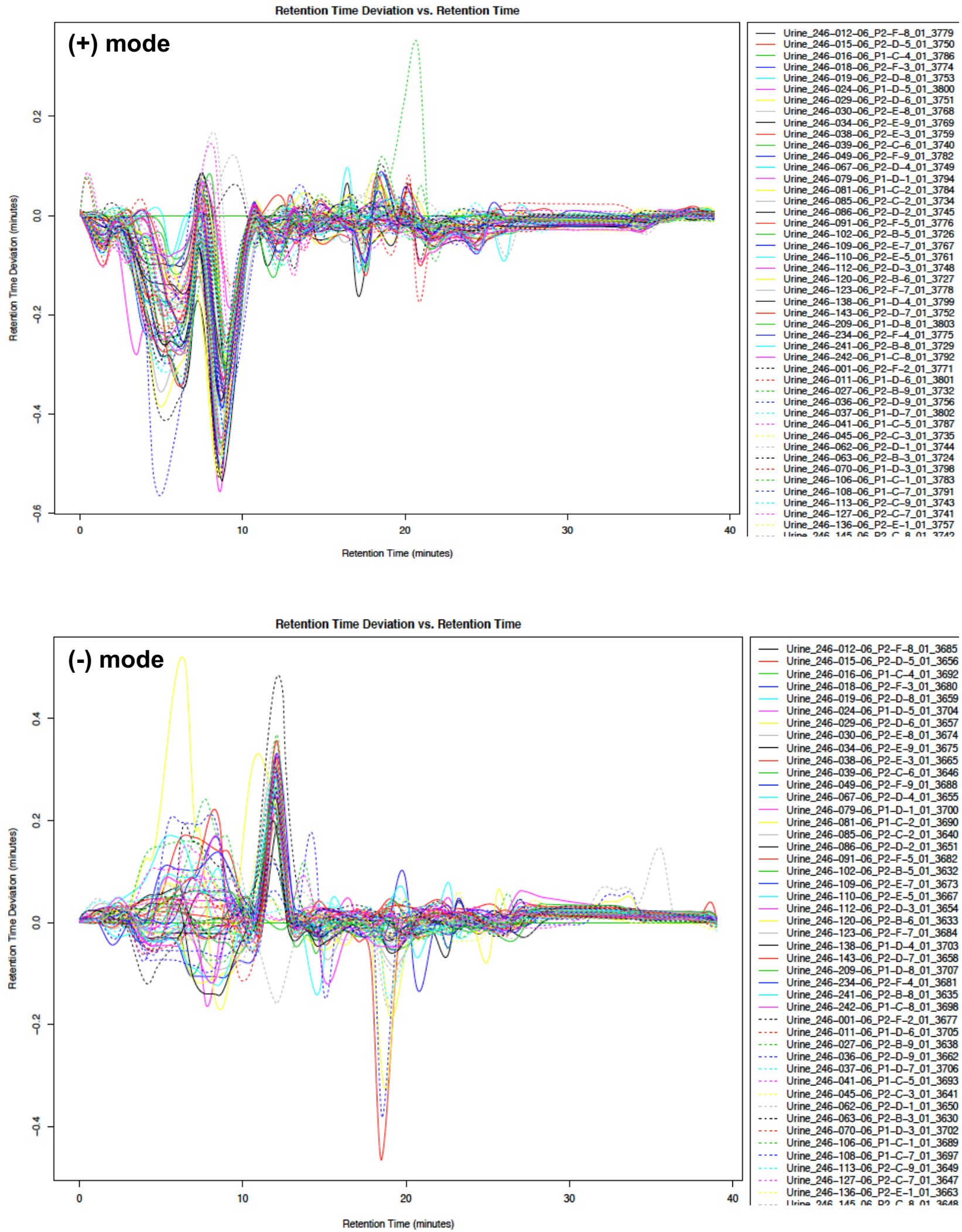

- Urine_246-012-06_P2-F-8_01_3685 - Urine_246-015-06_P2-D-5_01_3656 - Urine_246-016-06_P1-C-4_01_3692 - Urine_246-018-06_P2-F-3_01_3680 Urine_246-019-06_P2-D-8_01_3659 Urine_246-024-06_P1-D-5_01_3704 Urine_246-029-06_P2-D-6_01_3657 Urine_246-030-06_P2-E-8_01_3674 - Urine_246-034-06_P2-E-9_01_3675 - Urine_246-038-06_P2-E-3_01_3665 - Urine_246-049-06_P2-F-9_01_3688 - Urine_246-067-06_P2-D-4_01_3655

- Urine_246-079-06_P1-D-1_01_3700 Urine_246-081-06_P1-C-2_01_3690 Urine_246-085-06_P2-C-2_01_3640 - Urine_246-086-06_P2-D-2_01_3651 - Urine_246-091-06_P2-F-5_01_3682 - Urine_246-102-06_P2-B-5_01_3632 - Urine_246-109-06_P2-E-7_01_3673 - Urine_246-110-06_P2-E-5_01_3667 - Urine_246-112-06_P2-D-3_01_3654 Urine_246-120-06_P2-B-6_01_3633 Urine_246-123-06_P2-F-7_01_3684

- Urine_246-138-06_P1-D-4_01_3703 - Urine_246-143-06_P2-D-7_01_3658 - Urine_246-209-06_P1-D-8_01_3707 - Urine_246-241-06_P2-B-8_01_3635 - Urine_246-242-06_P1-C-8_01_3698 .... Urine_246-001-06_P2-F-2_01_3677 ... Urine_246-011-06_P1-D-6_01_3705 ... Urine_246-027-06_P2-B-9_01_3638 ...- Urine_246-036-06_P2-D-9_01_3662 Urine_246-037-06_P1-D-7_01_3706 Urine_246-041-06_P1-C-5_01_3693 Urine_246-045-06_P2-C-3_01_3641 Urine_246-062-06_P2-D-1_01_3650 Urine_246-063-06_P2-B-3_01_3630 Urine_246-070-06_P1-D-3_01_3702 Urine_246-106-06_P1-C-1_01_3689 Urine 246-108-06_P1-C-7_01_3697 Urine_246-113-06_P2-C-9_01_3649 Urine_246-113-06_P2-C-9_01_3649 Urine_246-136-06_P2-E-1_01_3663

Retention Time (minutes)

Fig. S2. Retention time deviation versus retention time of the 60 samples in positive (top panel) and negative mode data acquisition (bottom panel). 
Figure S3

(a) total cotinine
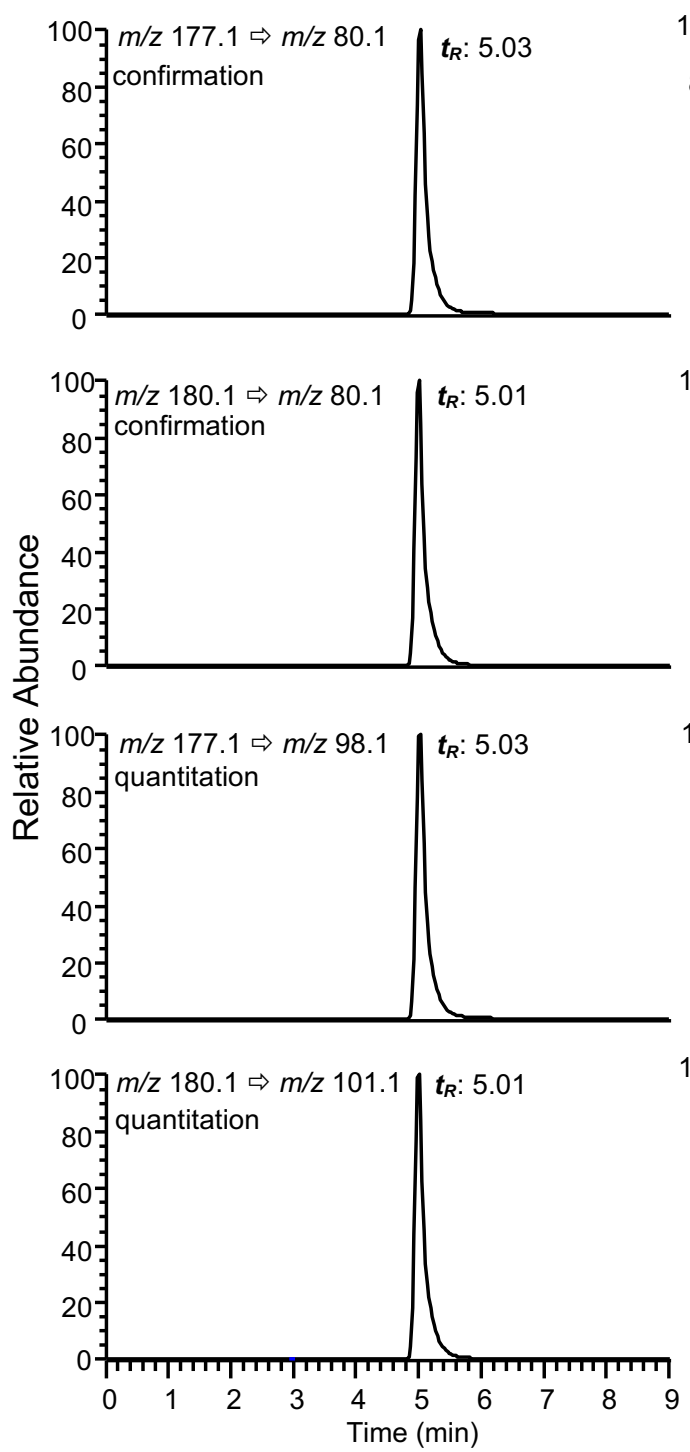

total trans-3-hydroxycotinine
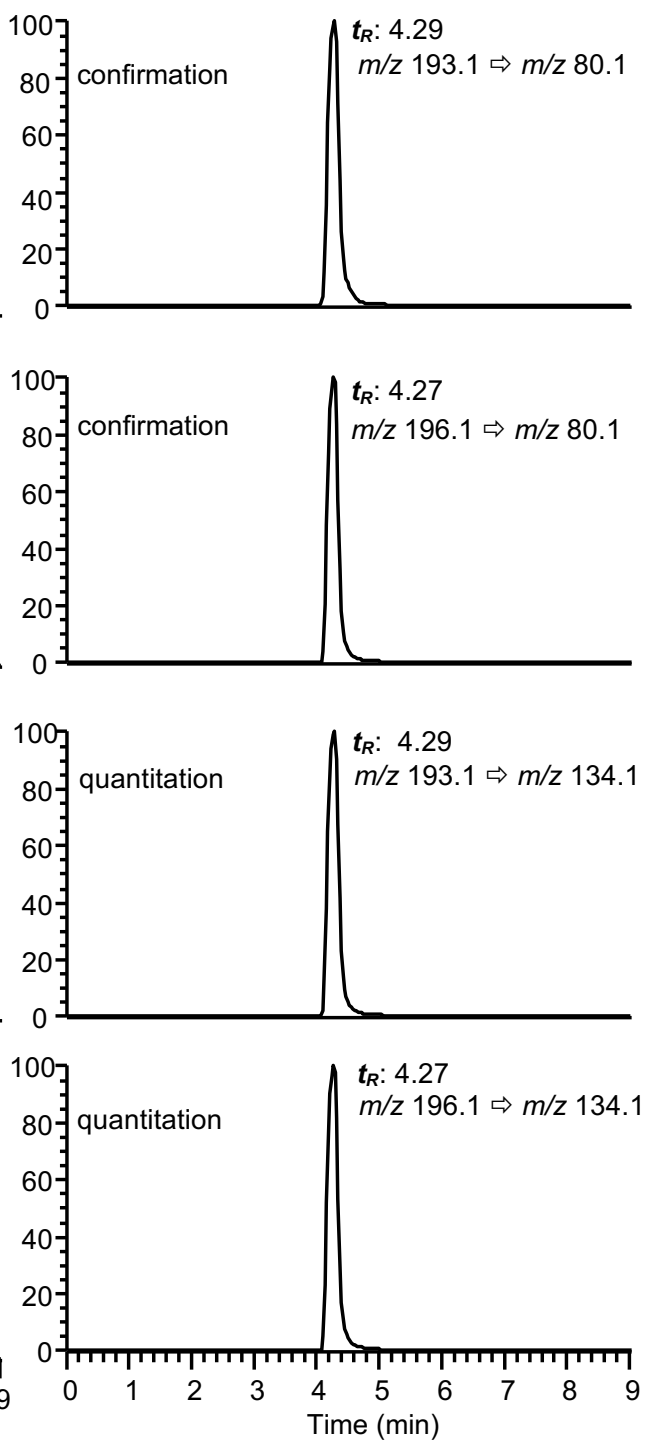


\section{Figure S3 (cont.)}

(b) free cotinine
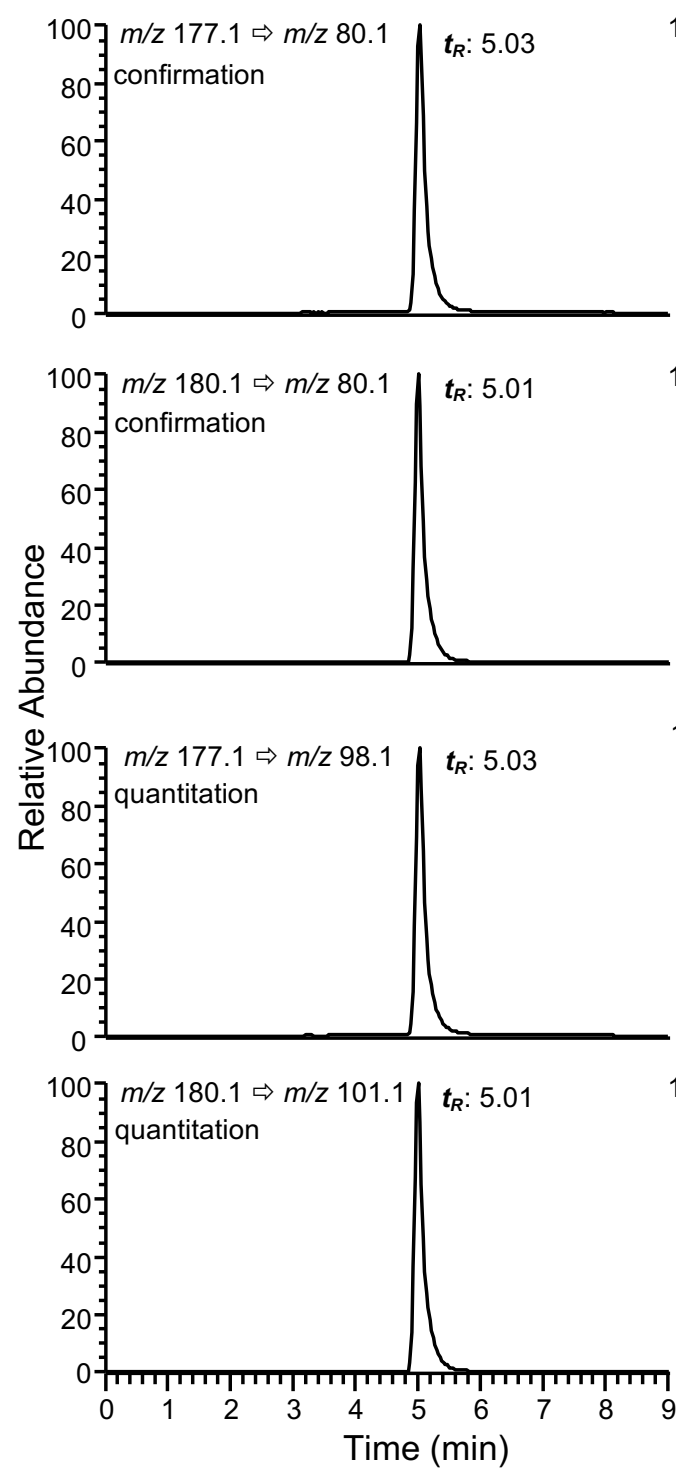

free trans-3-hydroxycotinine
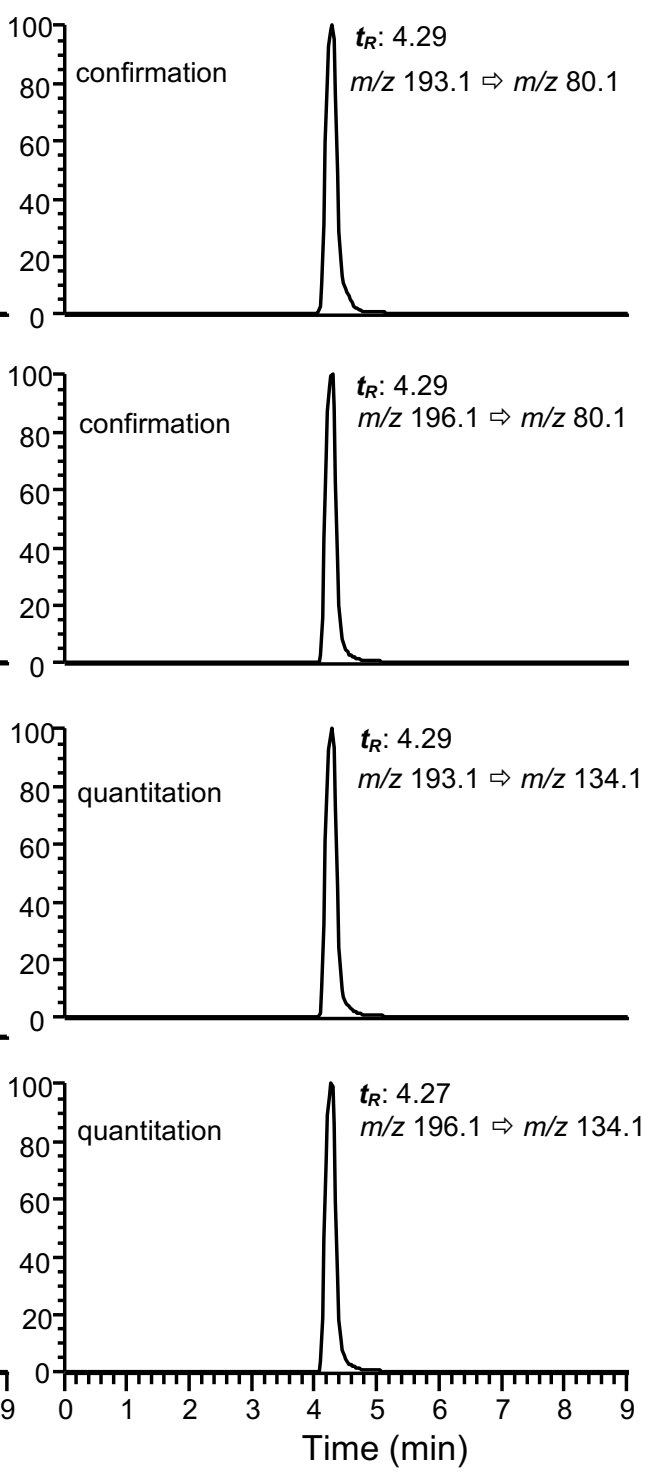

Fig. S3. (a) Extracted ion chromatograms (EICs) of the SRM transitions of total cotinine and trans3-hydroxycotinine; (b) EICs of the SRM transitions of free cotinine and trans-3-hydroxycotinine. 
Figure 54

(a) total cotinine

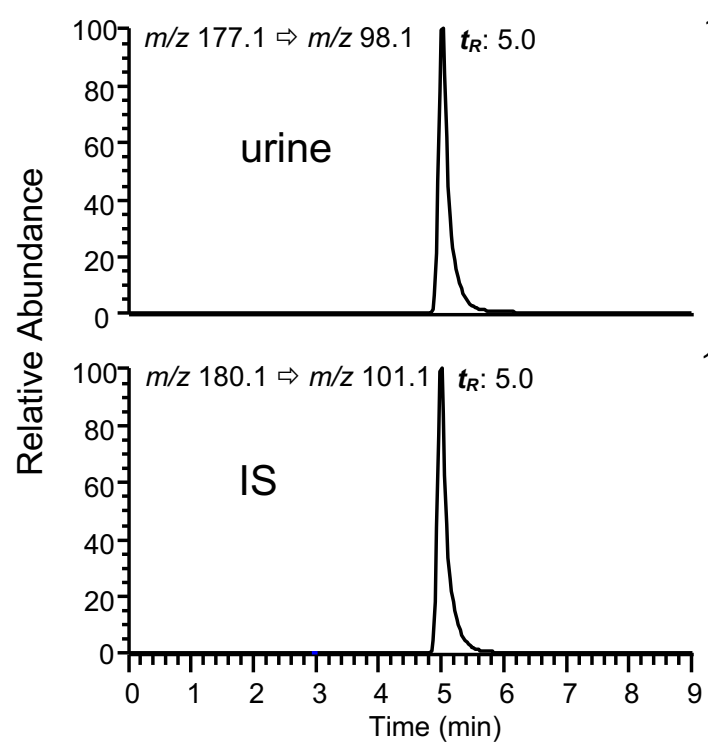

(b)

free cotinine

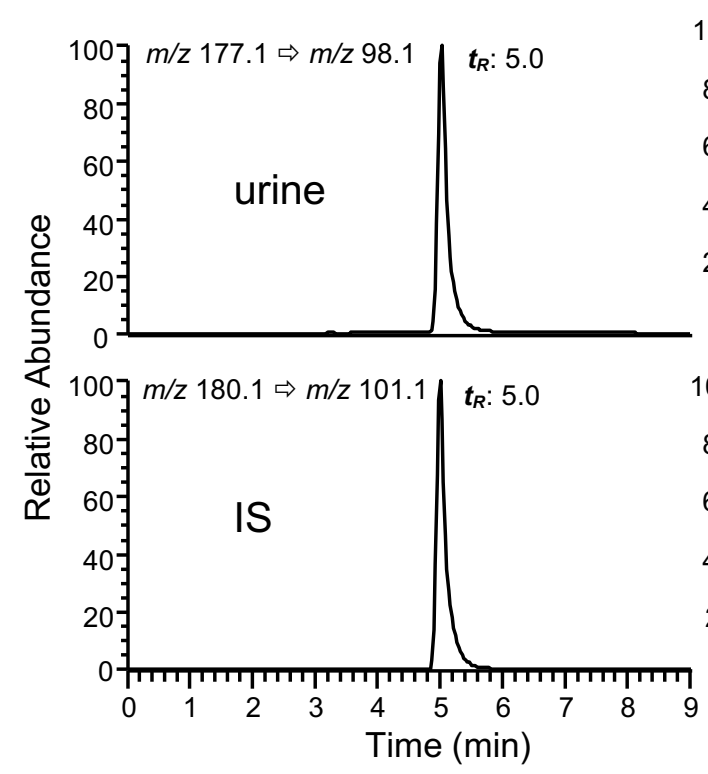

total trans-3-hydroxycotinine

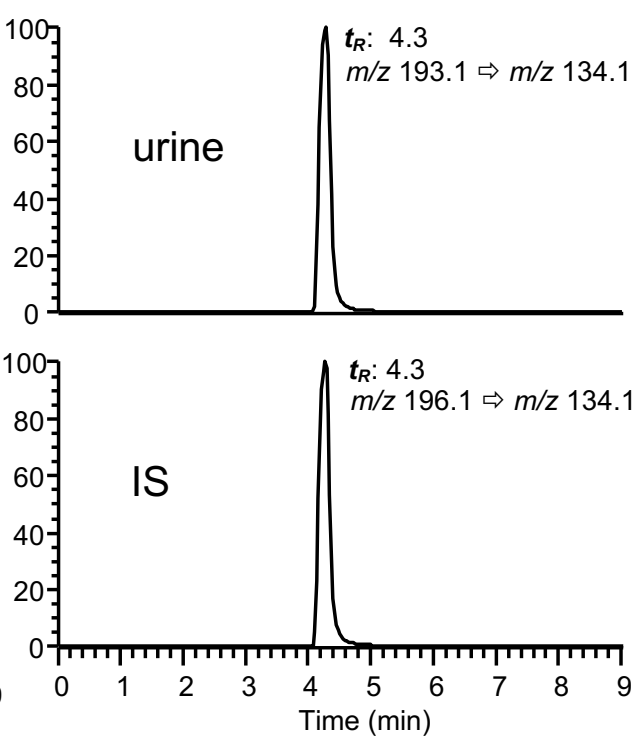

free trans-3-hydroxycotinine

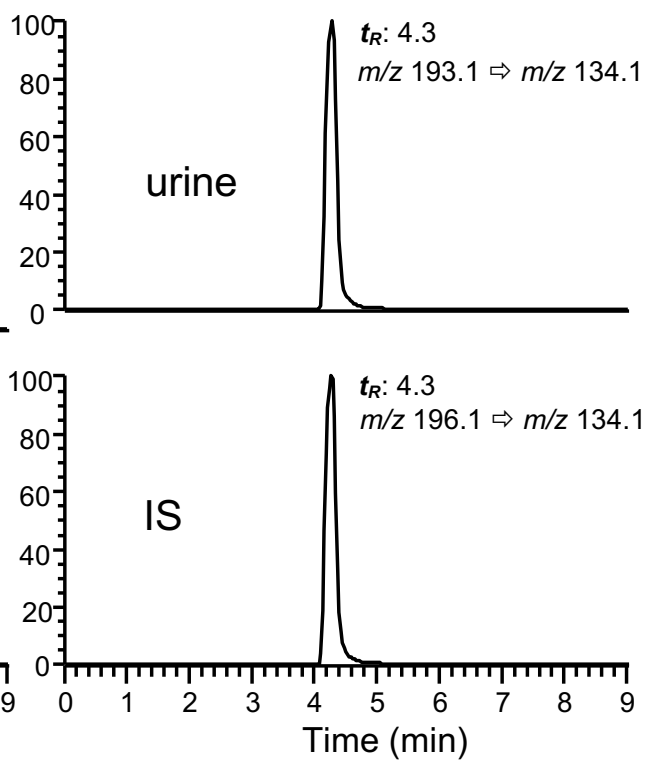

Fig. S4. Representative EICs of the SRM transitions of (a) total cotinine and trans-3hydroxycotinine; (b) free cotinine and trans-3-hydroxycotinine in urine samples with corresponding $d_{3}$-labeled IS. 
Figure S5
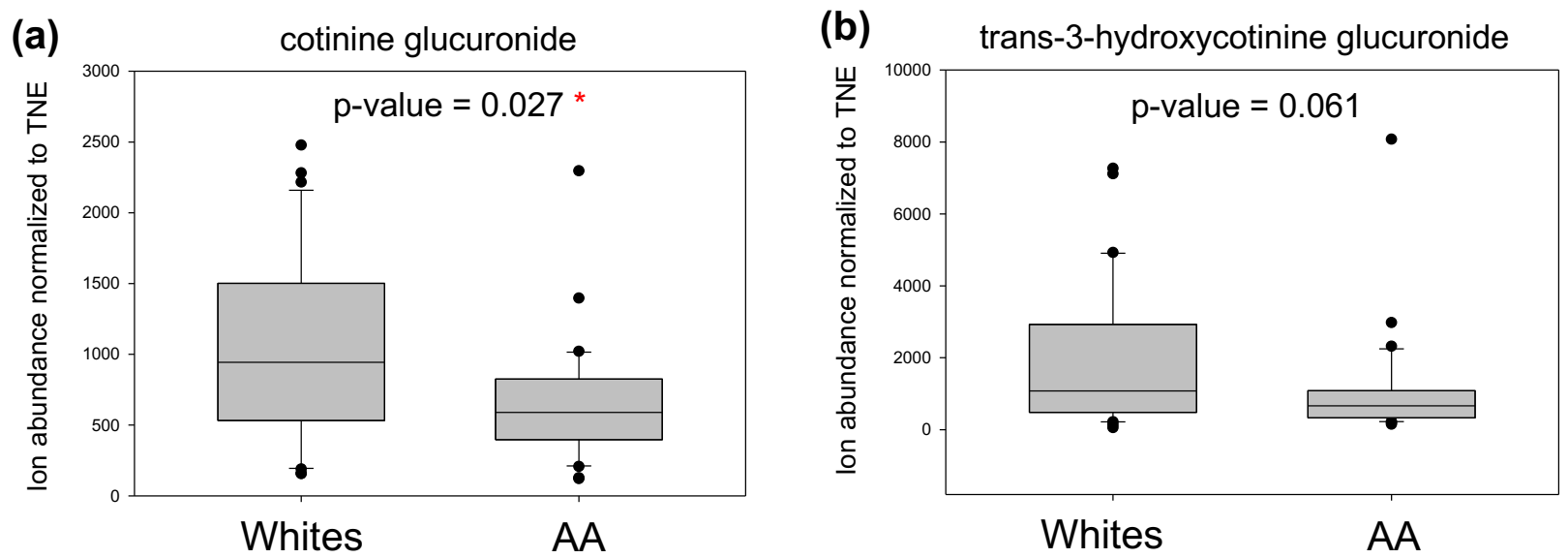

Fig. S5. (a) Ion abundance of cotinine glucuronide adjusted to TNE; (b) lon abundance of trans-3hydroxycotinine glucuronide adjusted to TNE in the metabolomics analysis. 


\section{Figure S6}

\section{(a) L-xylulose}
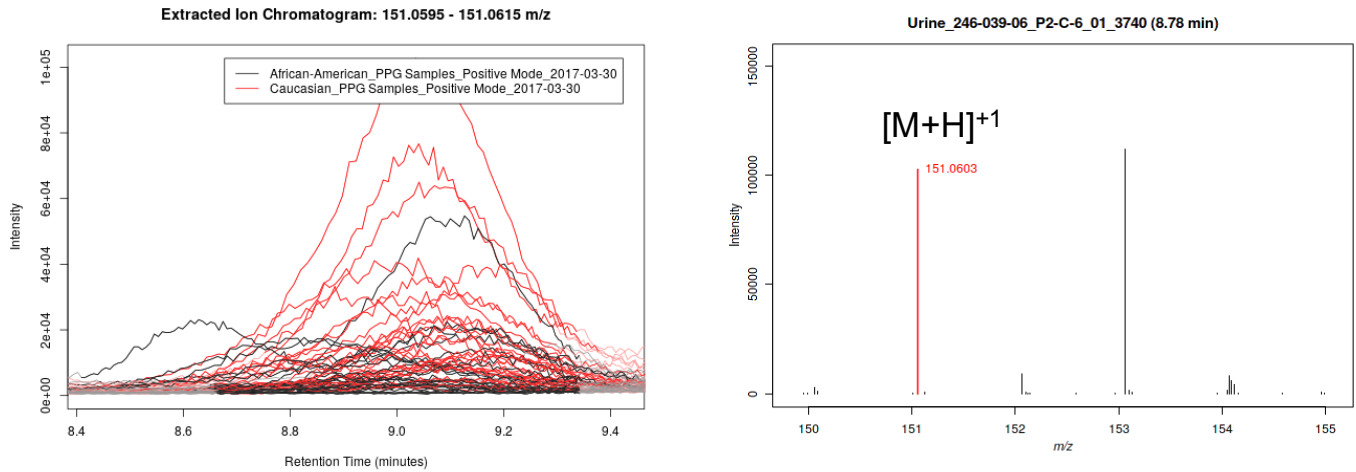

(b) L-gulonate
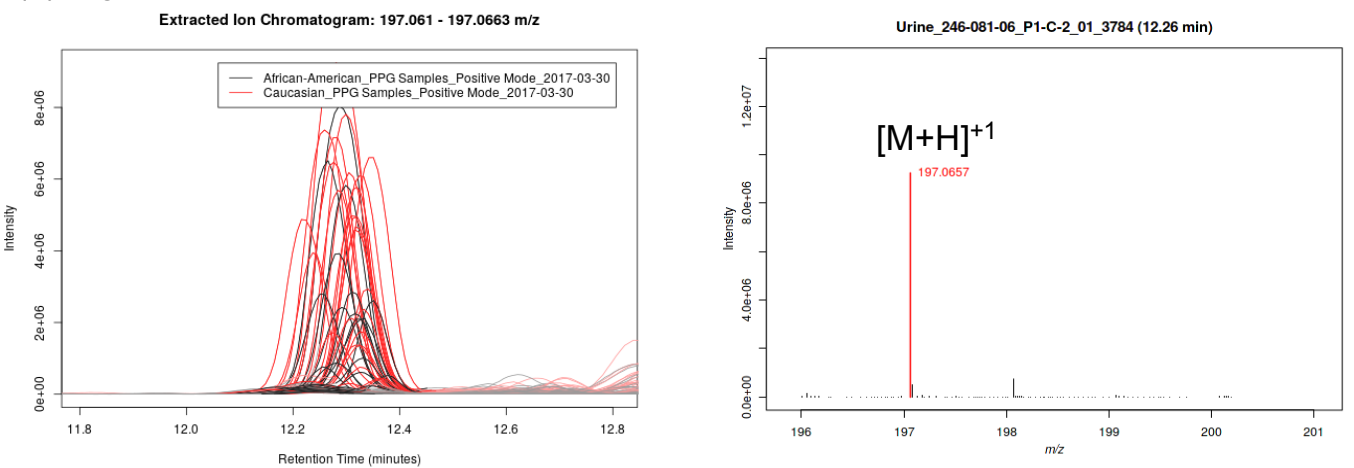

(c) aldehydo-D-glucuronate/3-keto-L-gulonate
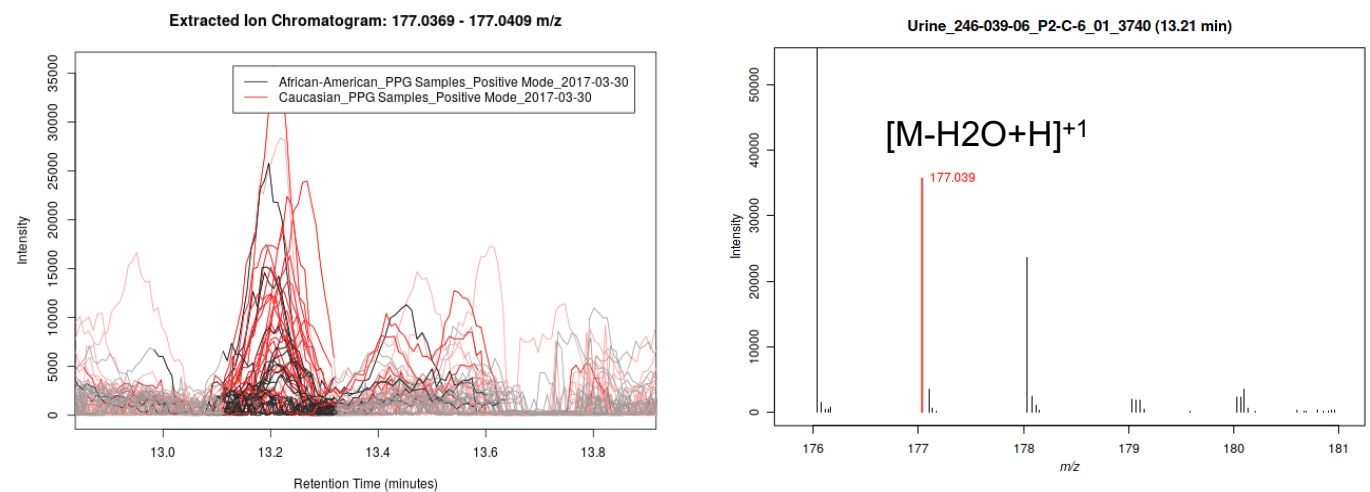

(d) 3-pyridylacetate
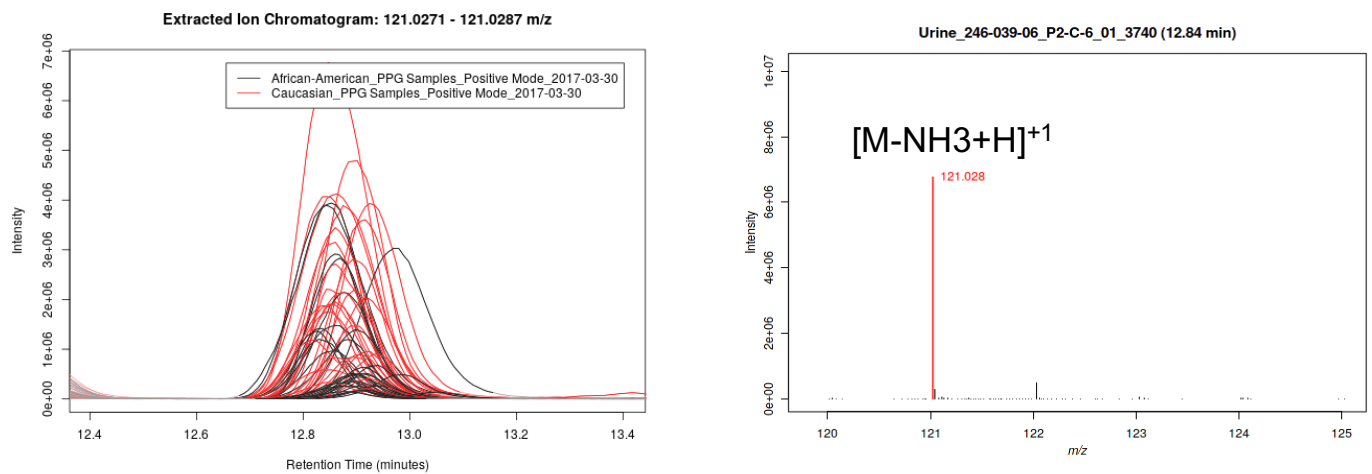


\section{Figure $\mathbf{S 6}$ (cont.)}

(e) 4-(3-pyridyl)-butanoate
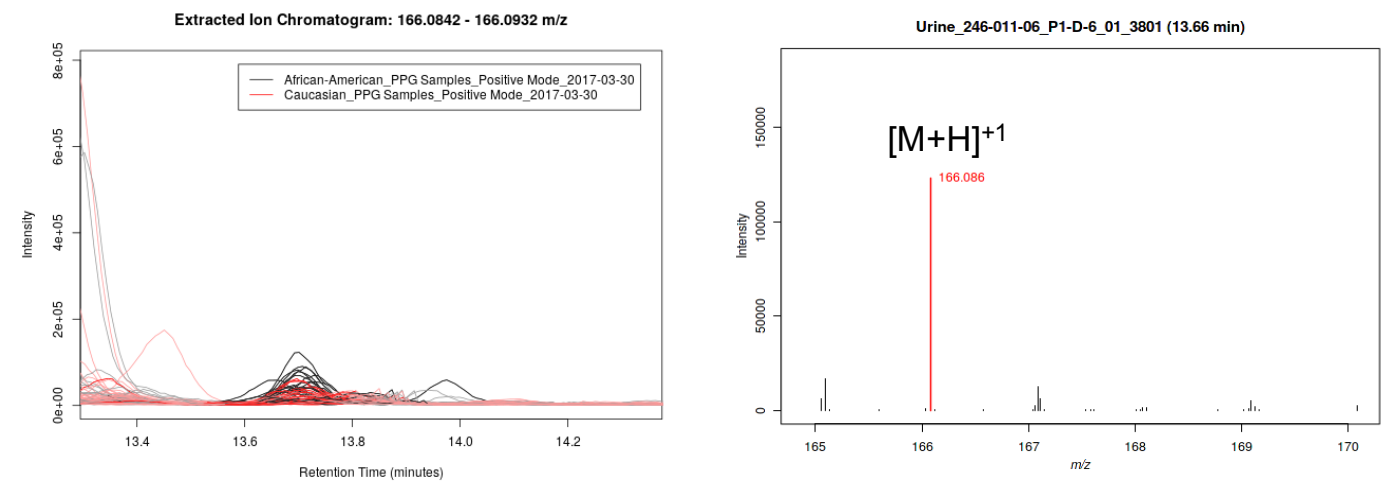

(f) cotinine methonium ion
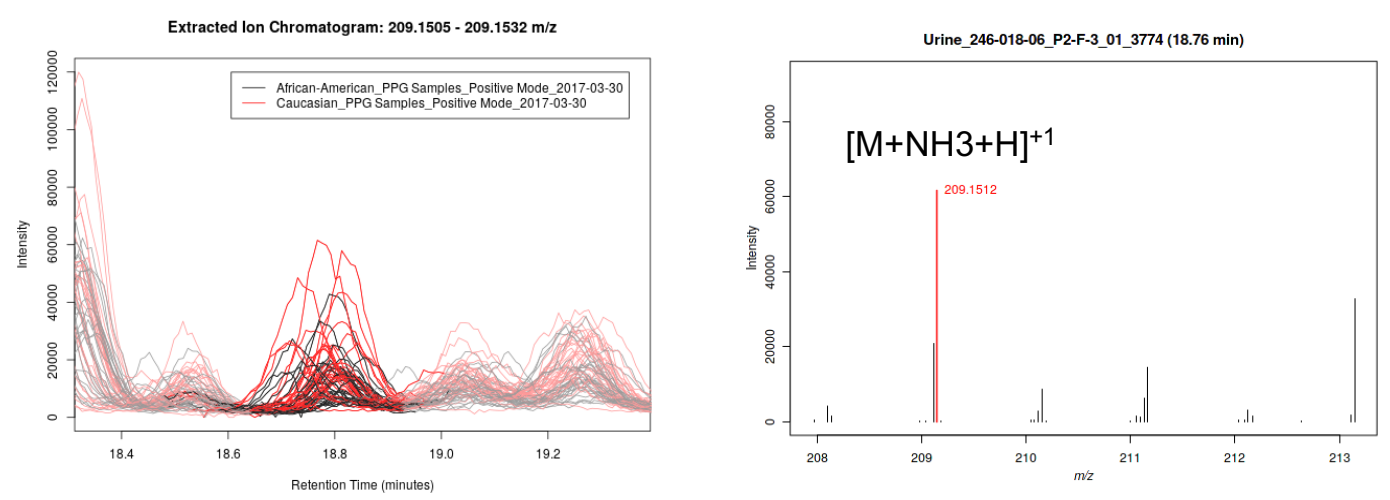

(g) trans-3-hydroxycotinine-glucuronide
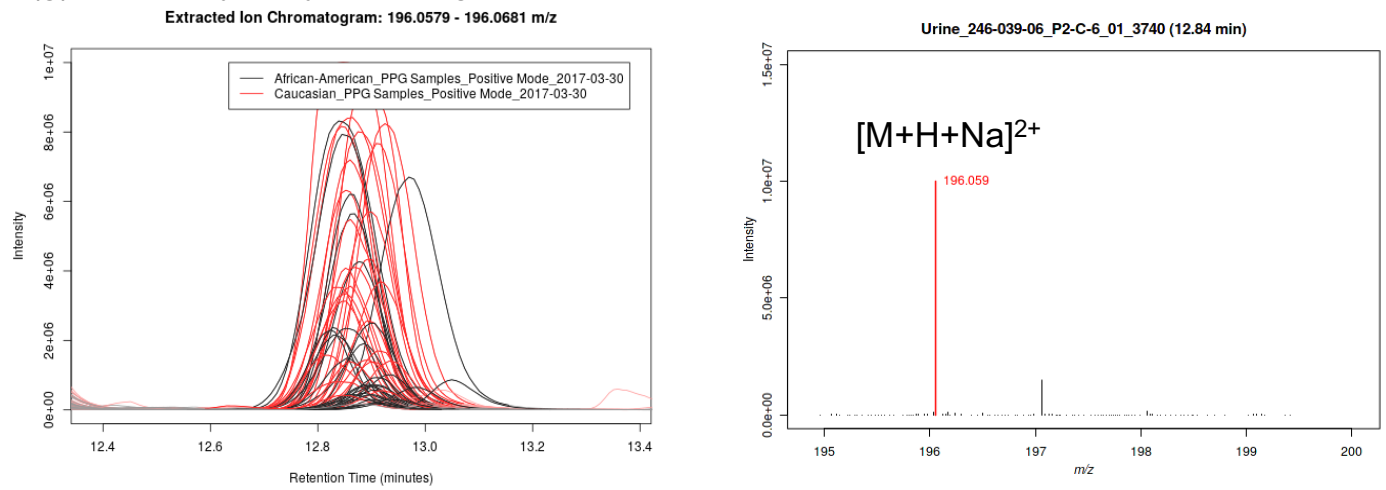

(h) cotinine-glucuronide
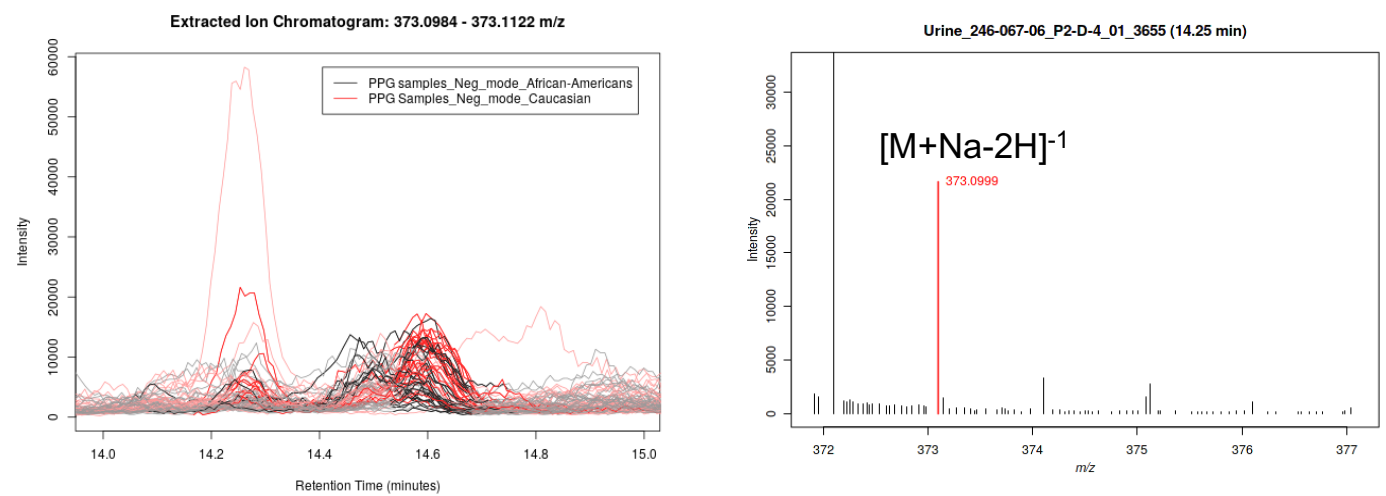


\section{Figure $\mathbf{S 6}$ (cont.)}

\section{(i) palmitate}

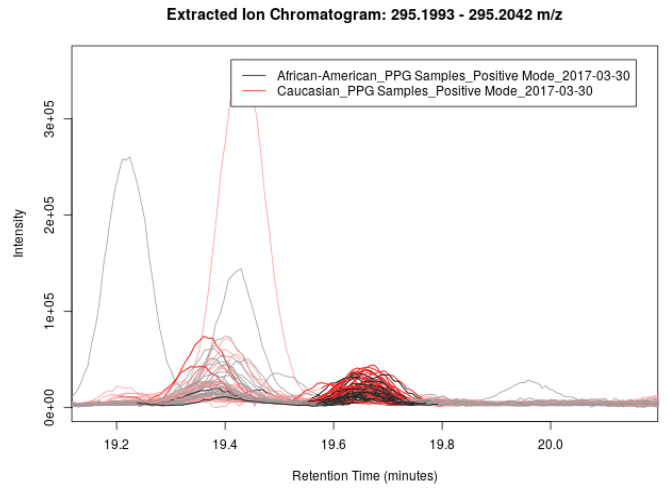

(j) L-lysine

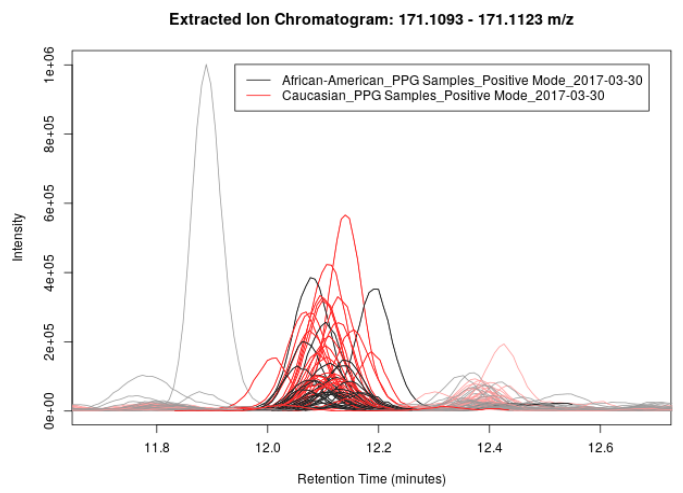

(k) L-pipecolate

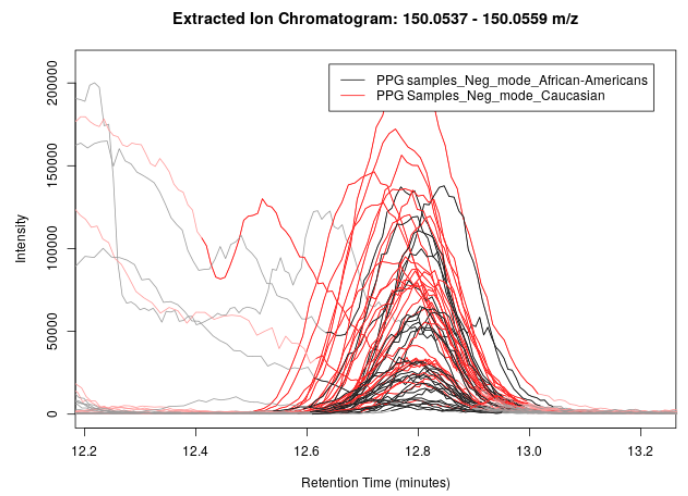

(I) L-histidine

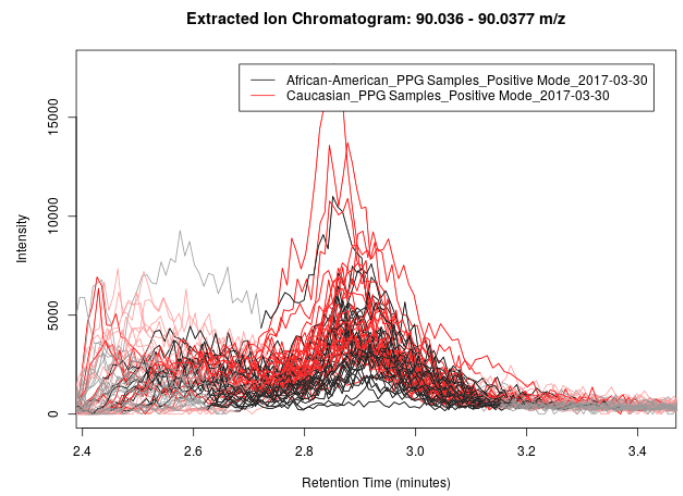

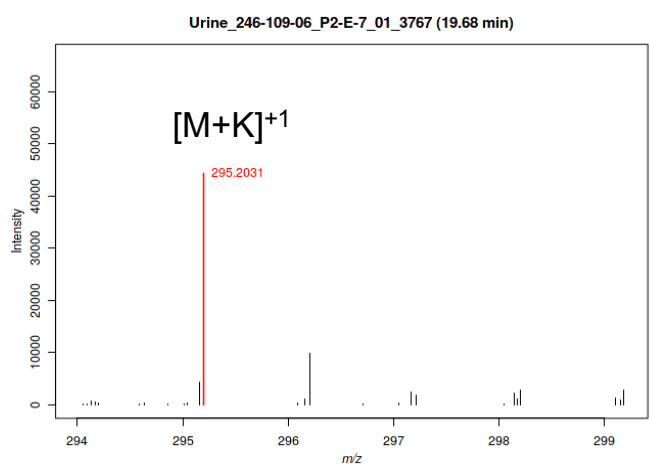
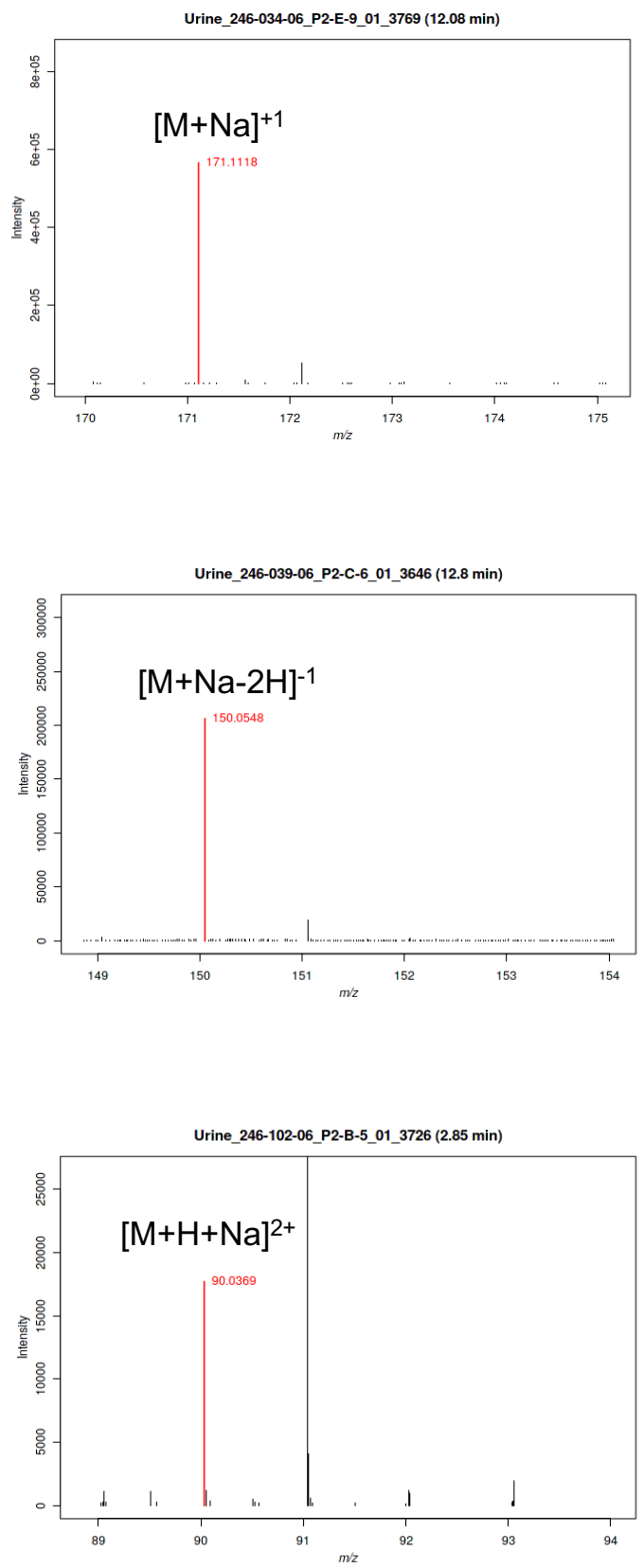


\section{Figure $\mathbf{S 6}$ (cont.)}

(m) (R)-adrenaline
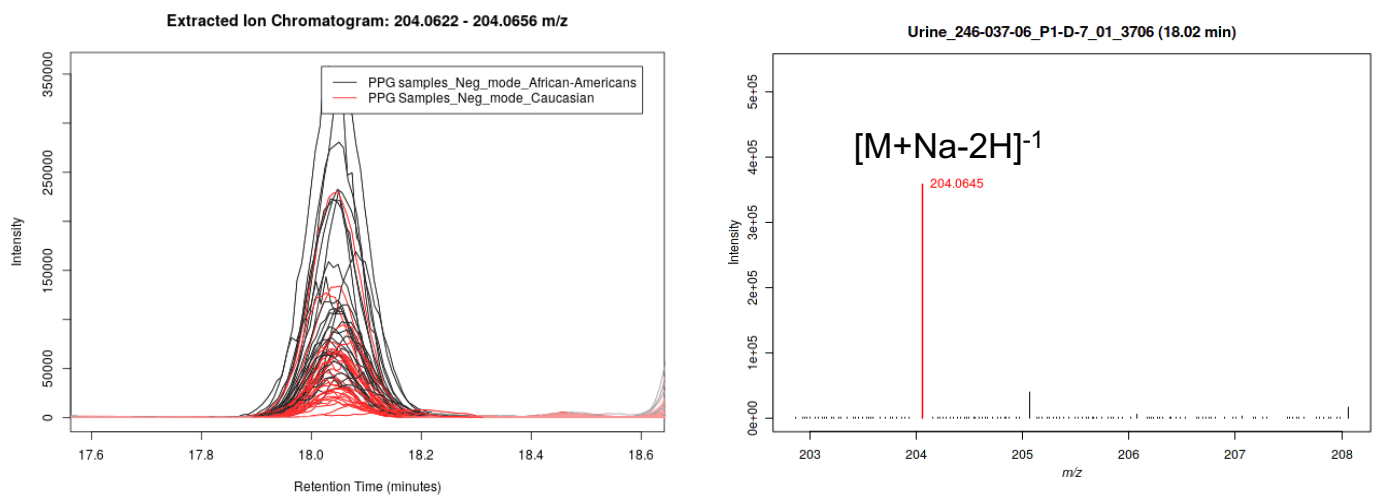

(n) 4a-hydroxy-tetrahydrobiopterin
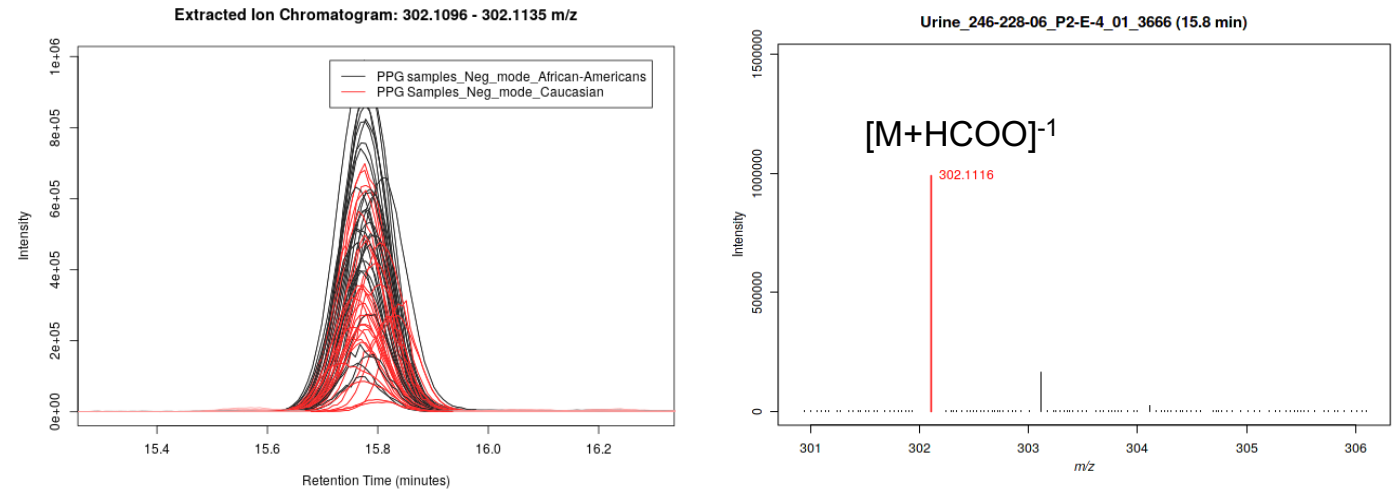

(o) L-ascorbate
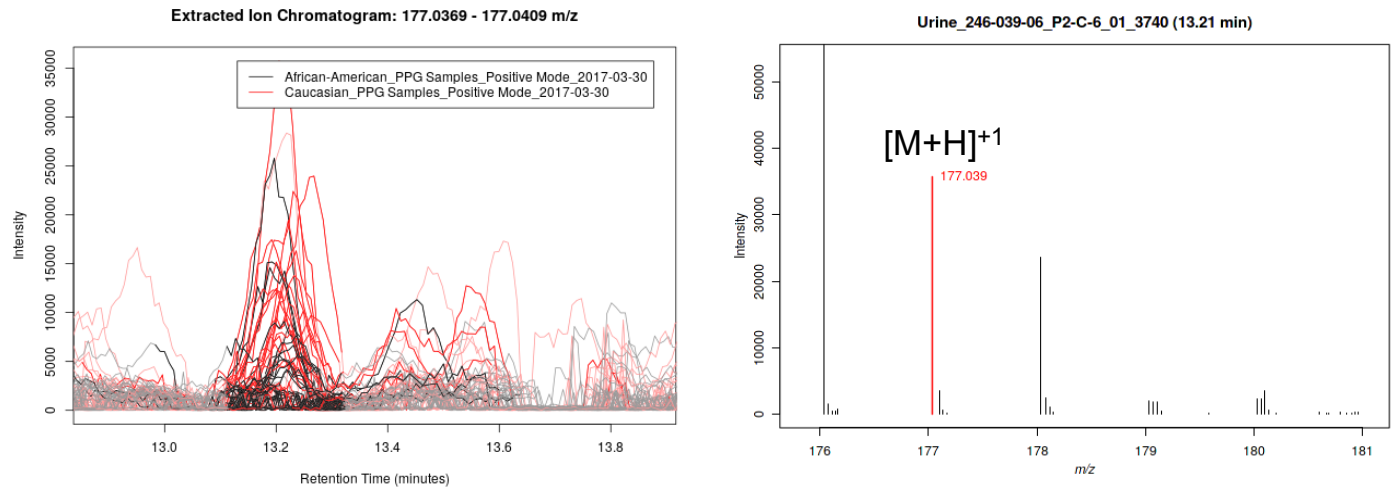

(p) $\beta$-D-glucose/ $\alpha$-D-glucose/ $\beta$-D-fructofuranose/ $\beta$-D-galactose/a-D-galactose
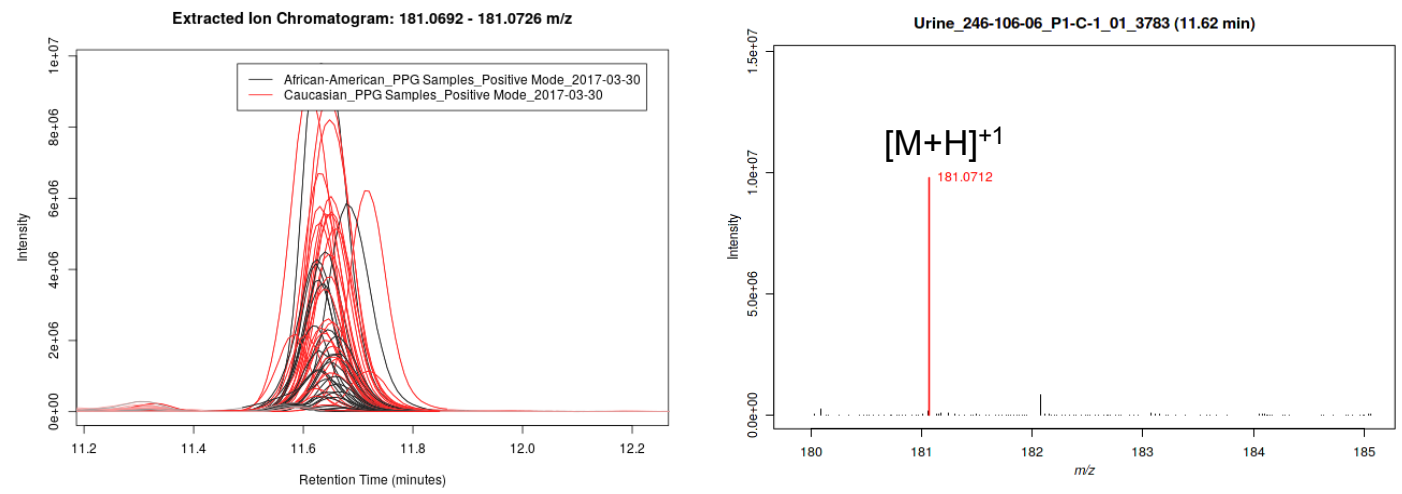


\section{Figure $\mathbf{S 6}$ (cont.)}

(q) oleate
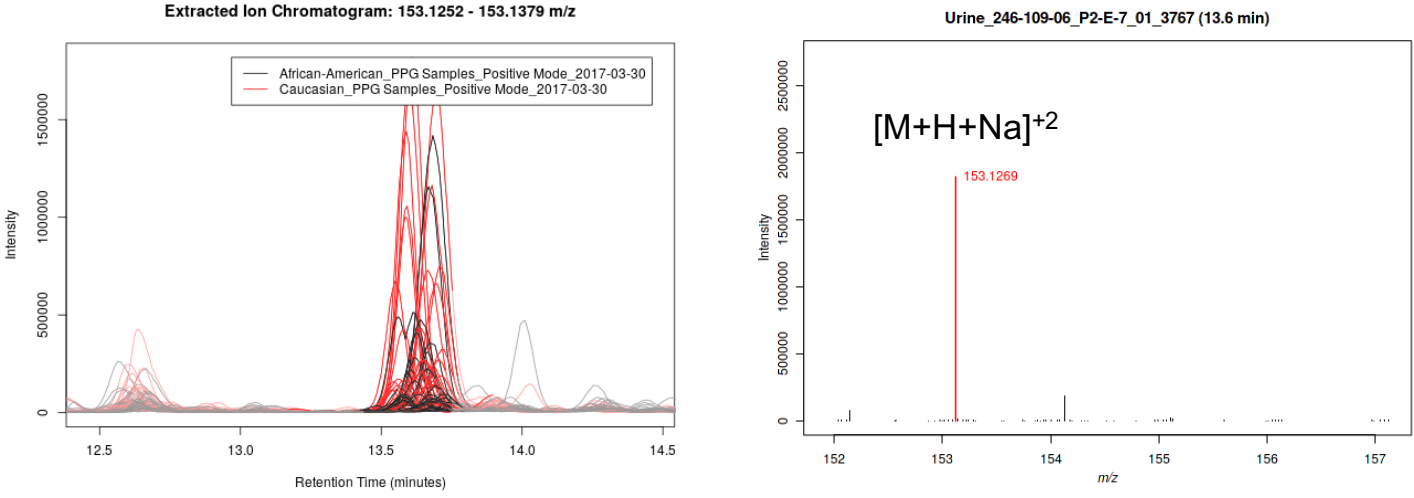

Fig. S6. Representative extracted ion chromatograms (EIC) and zoomed precursor ion full scan MS spectra of putative metabolites associated with differentially regulated pathways identified in the multi-modal pathway analysis. 
Table S1. Demographics of subjects, NNAL levels, and nicotine metabolites data.

\begin{tabular}{|c|c|c|c|c|c|c|c|c|c|}
\hline Subject & $\begin{array}{c}\text { TNE } \\
(\mathrm{nmol} / \mathrm{mL})\end{array}$ & $\begin{array}{c}\text { Creatinine } \\
(\mathrm{mg} / \mathrm{mL})\end{array}$ & $\begin{array}{c}\text { NNAL } \\
\text { (pg/mL) }\end{array}$ & Age & $\begin{array}{c}\text { Total COT } \\
\text { (ng/mL) }\end{array}$ & $\begin{array}{c}\text { Total } \\
\text { 3-HCOT } \\
\text { (ng/mL) }\end{array}$ & $\begin{array}{c}\text { Free COT } \\
\text { (ng/mL) }\end{array}$ & $\begin{array}{c}\text { Free } \\
\text { 3-HCOT } \\
\text { (ng/mL) }\end{array}$ & $\begin{array}{c}\text { COT-gluc } \\
\text { (ng/mL) }\end{array}$ \\
\hline \multicolumn{10}{|l|}{$\begin{array}{c}\text { African } \\
\text { American }\end{array}$} \\
\hline S001 & 59 & 1.9 & 106 & 50 & 4519 & 2798 & 1674 & 2128 & 2845 \\
\hline S002 & 83 & 1.5 & 158 & 45 & 4880 & 3411 & 2554 & 3097 & 2326 \\
\hline S003 & 67 & 1.1 & 167 & 46 & 2617 & 2894 & 1668 & 2365 & 950 \\
\hline S004 & 113 & 2.5 & 317 & 50 & 3836 & 7838 & 2102 & 5572 & 1734 \\
\hline S005 & 102 & 1.5 & 213 & 53 & 5034 & 15019 & 4789 & 12846 & 245 \\
\hline S006 & 89 & 1.8 & 285 & 57 & 3604 & 9790 & 1844 & 5582 & 1761 \\
\hline S007 & 32 & 0.7 & 151 & 50 & 2064 & 1463 & 1609 & 1031 & 455 \\
\hline S008 & 36 & 1.2 & 192 & 52 & 1484 & 7788 & 991 & 4500 & 493 \\
\hline S009 & 54 & 0.7 & 125 & 40 & 2508 & 3477 & 1608 & 2417 & 900 \\
\hline S010 & 51 & 1.4 & 125 & 33 & 3201 & 7071 & 2425 & 6265 & 776 \\
\hline S011 & 102 & 1.2 & 298 & 43 & 5103 & 18300 & 4425 & 12057 & 678 \\
\hline S012 & 130 & 2.2 & 242 & 52 & 4053 & 18198 & 2709 & 12989 & 1344 \\
\hline S013 & 75 & 1.0 & 152 & 44 & 5467 & 11218 & 2651 & 7514 & 2816 \\
\hline S014 & 80 & 1.1 & 206 & 50 & 7968 & 6655 & 2008 & 5355 & 5961 \\
\hline S015 & 83 & 0.8 & 340 & 50 & 2403 & 11138 & 1313 & 9045 & 1090 \\
\hline S016 & 50 & 0.6 & 164 & 50 & 3382 & 10100 & 3226 & 8176 & 155 \\
\hline S017 & 159 & 1.2 & 380 & 49 & 11671 & 26943 & 3756 & 20744 & 7915 \\
\hline S018 & 93 & 1.8 & 325 & 41 & 6064 & 16659 & 3337 & 11567 & 2727 \\
\hline S019 & 68 & 1.4 & 313 & 29 & 3728 & 8669 & 3259 & 7612 & 470 \\
\hline S020 & 100 & 1.0 & 304 & 44 & 3677 & 1225 & 2045 & 687 & 1632 \\
\hline S021 & 77 & 1.8 & 490 & 49 & 7473 & 12778 & 4364 & 10122 & 3109 \\
\hline S022 & 30 & 0.4 & 140 & 49 & 2382 & 2545 & 1703 & 2175 & 679 \\
\hline S023 & 33 & 1.0 & 129 & 43 & 1669 & 5159 & 618 & 4020 & 1051 \\
\hline S024 & 47 & 1.0 & 102 & 54 & 3341 & 5927 & 1826 & 4728 & 1515 \\
\hline S025 & 28 & 1.6 & 60 & 28 & 1432 & 4665 & 1236 & 2566 & 196 \\
\hline S026 & 51 & 1.1 & 114 & 54 & 2889 & 5478 & 1408 & 4458 & 1481 \\
\hline S027 & 91 & 1.8 & 257 & 54 & 5184 & 9870 & 2455 & 6262 & 2729 \\
\hline S028 & 69 & 3.5 & 367 & 40 & 5947 & 7893 & 3671 & 5879 & 2276 \\
\hline S029 & 60 & 1.6 & 91 & 55 & 2763 & 13200 & 1631 & 8504 & 1132 \\
\hline S030 & 77 & 1.5 & 410 & 26 & 5653 & 8781 & 3052 & 5944 & 2601 \\
\hline
\end{tabular}

White

\begin{tabular}{|c|c|c|c|c|c|c|c|c|c|}
\hline S031 & 53 & 1.3 & 132 & 52 & 3624 & 6591 & 1429 & 4491 & 2195 \\
\hline S032 & 69 & 0.4 & 215 & 62 & 4172 & 12031 & 2483 & 8933 & 1689 \\
\hline S033 & 124 & 1.3 & 467 & 58 & 8103 & 18639 & 2499 & 12177 & 5604 \\
\hline S034 & 95 & 1.0 & 153 & 20 & 8594 & 10321 & 2624 & 7898 & 5970 \\
\hline S035 & 30 & 2.5 & 193 & 18 & 1665 & 4961 & 794 & 4273 & 871 \\
\hline S036 & 79 & 1.6 & 58 & 26 & 6616 & 3809 & 1975 & 2693 & 4641 \\
\hline S037 & 70 & 1.3 & 219 & 21 & 8278 & 18385 & 2217 & 14307 & 6062 \\
\hline S038 & 98 & 1.9 & 319 & 31 & 8412 & 12639 & 2430 & 9856 & 5982 \\
\hline S039 & 77 & 1.7 & 157 & 30 & 7139 & 12240 & 2581 & 8089 & 4558 \\
\hline S040 & 47 & 0.9 & 179 & 26 & 2379 & 8787 & 1221 & 7088 & 1159 \\
\hline S041 & 131 & 1.3 & 314 & 51 & 5456 & 19850 & 2985 & 16222 & 2472 \\
\hline S042 & 95 & 1.4 & 276 & 54 & 4237 & 17637 & 1544 & 12718 & 2694 \\
\hline S043 & 90 & 1.5 & 179 & 42 & 5087 & 8346 & 1931 & 5985 & 3156 \\
\hline S044 & 76 & 0.5 & 159 & 36 & 6165 & 8487 & 2612 & 6157 & 3553 \\
\hline S045 & 29 & 0.5 & 108 & 43 & 1500 & 4660 & 898 & 3363 & 601 \\
\hline S046 & 173 & 2.3 & 545 & 45 & 9920 & 35479 & 2287 & 30064 & 7633 \\
\hline S047 & 153 & 1.9 & 357 & 28 & 8050 & 23859 & 3378 & 18891 & 4673 \\
\hline S048 & 73 & 1.5 & 309 & 36 & 2961 & 18336 & 2246 & 14788 & 715 \\
\hline S049 & 115 & 1.2 & 276 & 53 & 7661 & 9743 & 2574 & 8036 & 5086 \\
\hline S050 & 62 & 1.2 & 110 & 43 & 5075 & 6346 & 1652 & 5203 & 3424 \\
\hline S051 & 75 & 0.8 & 227 & 48 & 5270 & 11624 & 2382 & 7603 & 2887 \\
\hline S052 & 20 & 0.3 & 131 & 52 & 1078 & 785 & 607 & 638 & 471 \\
\hline S053 & 40 & 0.9 & 112 & 50 & 2239 & 4492 & 657 & 3185 & 1582 \\
\hline S054 & 132 & 1.2 & 337 & 37 & 8999 & 12145 & 2585 & 8587 & 6414 \\
\hline S055 & 74 & 1.1 & 178 & 24 & 4680 & 10503 & 1819 & 7528 & 2861 \\
\hline S056 & 107 & 1.5 & 389 & 47 & 6343 & 19174 & 1678 & 13373 & 4664 \\
\hline S057 & 53 & 0.8 & 61 & 41 & 1620 & 9067 & 1527 & 5580 & 93 \\
\hline S058 & 64 & 0.4 & 119 & 60 & 5801 & 6887 & 3037 & 5342 & 2764 \\
\hline S059 & 63 & 0.9 & 172 & 47 & 5629 & 11248 & 2183 & 8653 & 3445 \\
\hline S060 & 41 & 0.4 & 92 & 29 & 3176 & 6824 & 1212 & 5519 & 1965 \\
\hline
\end{tabular}


Table S2. Levels of nicotine metabolites normalized to TNE.

\begin{tabular}{|c|c|c|}
\hline (a) cotinine glucuronide & AVG & STDEV \\
\hline Whites $(n=30)$ & 40 & 18 \\
\hline $\mathrm{AA}(n=30)$ & 24 & 16 \\
\hline (b) trans-3-hydroxycotinine glucuronide & AVG & STDEV \\
\hline Whites $(n=30)$ & 36 & 15 \\
\hline $\mathrm{AA}(n=30)$ & 33 & 22 \\
\hline (c) free cotinine & AVG & STDEV \\
\hline Whites $(n=30)$ & 27 & 7 \\
\hline AA $(n=30)$ & 35 & 13 \\
\hline (d) free trans-3-hydroxycotinine & AVG & STDEV \\
\hline Whites $(n=30)$ & 109 & 41 \\
\hline $\mathrm{AA}(n=30)$ & 89 & 39 \\
\hline (e) total cotinine & AVG & STDEV \\
\hline Whites $(n=30)$ & 67 & 19 \\
\hline $\mathrm{AA}(n=30)$ & 59 & 19 \\
\hline (f) total trans-3-hydroxycotinine & AVG & STDEV \\
\hline Whites $(n=30)$ & 145 & 51 \\
\hline $\mathrm{AA}(n=30)$ & 122 & 55 \\
\hline
\end{tabular}


Table S3. Differentially regulated metabolic pathways determined in the autonomous multi-modal pathway analysis with $\mathrm{p}$-value $=0.01$ and $5 \mathrm{ppm}$ mass tolerance.

\begin{tabular}{|c|c|c|c|c|}
\hline Pathway & $\begin{array}{c}\text { Overlapping putative } \\
\text { metabolites }^{1}\end{array}$ & $\begin{array}{c}\% \text { Overlapping putative } \\
\text { metabolites } 1\end{array}$ & All metabolites ${ }^{2}$ & p-value \\
\hline D-glucuronate degradation & 5 & $83.3 \%$ & 6 & 0.0002 \\
\hline bupropion degradation & 4 & $80.0 \%$ & 5 & 0.0002 \\
\hline nicotine degradation $\mathrm{V}$ & 5 & $27.8 \%$ & 18 & 0.0002 \\
\hline sucrose degradation & 3 & $60.0 \%$ & 5 & 0.0002 \\
\hline lysine degradation I (saccharopine pathway) & 3 & $42.9 \%$ & 7 & 0.0002 \\
\hline lactose degradation III & 2 & $100.0 \%$ & 2 & 0.0002 \\
\hline D-galactose degradation V (Leloir pathway) & 2 & $100.0 \%$ & 2 & 0.0002 \\
\hline lysine degradation II (pipecolate pathway) & 3 & $30.0 \%$ & 10 & 0.0002 \\
\hline dermatan sulfate degradation (metazoa) & 2 & $66.7 \%$ & 3 & 0.0002 \\
\hline trehalose degradation & 2 & $66.7 \%$ & 3 & 0.0002 \\
\hline thyroid hormone metabolism II (via conjugation and/or degradation) & 3 & $75.0 \%$ & 4 & 0.0002 \\
\hline myo-inositol de novo biosynthesis & 1 & $100.0 \%$ & 1 & 0.0003 \\
\hline sulfite oxidation & 1 & $100.0 \%$ & 1 & 0.0003 \\
\hline oleate biosynthesis & 1 & $100.0 \%$ & 1 & 0.0003 \\
\hline D-myo-inositol $(1,4,5)$-trisphosphate biosynthesis & 1 & $100.0 \%$ & 1 & 0.0003 \\
\hline D-myo-inositol $(1,4,5)$-trisphosphate degradation & 1 & $100.0 \%$ & 1 & 0.0003 \\
\hline thiamin salvage III & 1 & $100.0 \%$ & 1 & 0.0003 \\
\hline glycogenolysis & 1 & $100.0 \%$ & 1 & 0.0003 \\
\hline 3-phosphoinositide biosynthesis & 1 & $100.0 \%$ & 1 & 0.0003 \\
\hline phosphatidylserine biosynthesis I & 1 & $100.0 \%$ & 1 & 0.0003 \\
\hline tRNA charging & 2 & $15.4 \%$ & 13 & 0.0003 \\
\hline a-tocopherol degradation & 1 & $50.0 \%$ & 2 & 0.0003 \\
\hline UDP-N-acetyl-D-galactosamine biosynthesis II & 1 & $50.0 \%$ & 2 & 0.0003 \\
\hline sorbitol degradation I & 1 & $50.0 \%$ & 2 & 0.0003 \\
\hline chondroitin sulfate degradation (metazoa) & 1 & $50.0 \%$ & 2 & 0.0003 \\
\hline sulfate activation for sulfonation & 1 & $50.0 \%$ & 2 & 0.0003 \\
\hline phospholipases & 1 & $50.0 \%$ & 2 & 0.0003 \\
\hline palmitate biosynthesis & 1 & $50.0 \%$ & 2 & 0.0003 \\
\hline histamine biosynthesis & 1 & $50.0 \%$ & 2 & 0.0003 \\
\hline stearate biosynthesis & 1 & $50.0 \%$ & 2 & 0.0003 \\
\hline carnosine biosynthesis & 1 & $50.0 \%$ & 2 & 0.0003 \\
\hline pyrimidine deoxyribonucleotide phosphorylation & 1 & $33.3 \%$ & 3 & 0.0004 \\
\hline pyrimidine deoxyribonucleotides biosynthesis from CTP & 1 & $33.3 \%$ & 3 & 0.0004 \\
\hline phosphatidylcholine biosynthesis & 1 & $33.3 \%$ & 3 & 0.0004 \\
\hline homocarnosine biosynthesis & 1 & $33.3 \%$ & 3 & 0.0004 \\
\hline nicotine degradation IV & 2 & $11.8 \%$ & 17 & 0.0004 \\
\hline hydrogen sulfide biosynthesis (trans-sulfuration) & 1 & $25.0 \%$ & 4 & 0.0005 \\
\hline choline degradation & 1 & $25.0 \%$ & 4 & 0.0005 \\
\hline tetrapyrrole biosynthesis & 1 & $25.0 \%$ & 4 & 0.0005 \\
\hline proline degradation & 1 & $20.0 \%$ & 5 & 0.0007 \\
\hline proline biosynthesis & 1 & $20.0 \%$ & 5 & 0.0007 \\
\hline ornithine de novo biosynthesis & 1 & $20.0 \%$ & 5 & 0.0007 \\
\hline pyrimidine deoxyribonucleotides de novo biosynthesis & 1 & $20.0 \%$ & 5 & 0.0007 \\
\hline histidine degradation & 2 & $40.0 \%$ & 5 & 0.0007 \\
\hline ascorbate recycling (cytosolic) & 1 & $20.0 \%$ & 5 & 0.0007 \\
\hline estradiol biosynthesis I & 1 & $20.0 \%$ & 5 & 0.0007 \\
\hline tryptophan degradation to 2-amino-3-carboxymuconate semialdehyde & 1 & $20.0 \%$ & 5 & 0.0007 \\
\hline thyronamine and iodothyronamine metabolism & 1 & $16.7 \%$ & 6 & 0.0009 \\
\hline adenine and adenosine salvage III & 2 & $33.3 \%$ & 6 & 0.0009 \\
\hline adenosine nucleotides degradation & 1 & $16.7 \%$ & 6 & 0.0009 \\
\hline 4-hydroxyproline degradation & 1 & $14.3 \%$ & 7 & 0.0012 \\
\hline purine ribonucleosides degradation to ribose-1-phosphate & 1 & $12.5 \%$ & 8 & 0.0017 \\
\hline serotonin degradation & 1 & $11.1 \%$ & 9 & 0.0022 \\
\hline androgen biosynthesis & 2 & $22.2 \%$ & 9 & 0.0022 \\
\hline catecholamine biosynthesis & 3 & $30.0 \%$ & 10 & 0.0029 \\
\hline
\end{tabular}

1) Number of significantly dysregulated metabolites found in pathway

2) Total number of putative metabolites found in the pathway using the entire LC-MS analysis results 
Table S4. Differentially regulated metabolic pathways and the associated metabolites determined in the autonomous multi-modal pathway analysis ( $p$-value $=0.01$ and 5 ppm mass tolerance).

\begin{tabular}{|c|c|c|c|c|c|c|c|}
\hline Pathway & METLIN ID & Dysregulation* & $\begin{array}{c}\text { Fold } \\
\text { Change }\end{array}$ & p-value & $m / z$ & $t_{R}(\min )$ & Adduct Form \\
\hline \multicolumn{8}{|l|}{ D-glucuronate degradation } \\
\hline L-xylulose & 139 & DOWN & 2.2 & $3.00 \mathrm{E}-03$ & 151.0604 & 9.08 & $\mathrm{M}+\mathrm{H}[1+]$ \\
\hline L-gulonate & 63144 & DOWN & 2.4 & $2.40 \mathrm{E}-03$ & 197.0652 & 12.29 & $\mathrm{M}+\mathrm{H}[1+]$ \\
\hline L-gulonate & 63144 & DOWN & 3 & $1.50 \mathrm{E}-04$ & 195.0503 & 11.99 & $\mathrm{M}-\mathrm{H}[-]$ \\
\hline aldehydo-D-glucuronatea & NA & DOWN & 2 & $1.70 \mathrm{E}-03$ & 177.0392 & 13.23 & $\mathrm{M}-\mathrm{H} 2 \mathrm{O}+\mathrm{H}[1+]$ \\
\hline 3-keto-L-gulonatea & 58394 & DOWN & 2 & $1.70 \mathrm{E}-03$ & 177.0392 & 13.23 & $\mathrm{M}-\mathrm{H} 2 \mathrm{O}+\mathrm{H}[1+]$ \\
\hline \multicolumn{8}{|l|}{ nicotine degradation $\mathbf{V} b$} \\
\hline 3-pyridylacetate & NA & DOWN & 2.1 & $4.50 \mathrm{E}-03$ & 121.0279 & 12.88 & $\mathrm{M}-\mathrm{NH} 3+\mathrm{H}[1+]$ \\
\hline 4-(3-pyridyl)-butanoate & NA & UP & 1.7 & $6.60 \mathrm{E}-03$ & 166.0855 & 13.71 & $\mathrm{M}+\mathrm{H}[1+]$ \\
\hline cotinine-glucuronide & NA & DOWN & 1.5 & 7.20E-03 & 373.1022 & 14.59 & $\mathrm{M}+\mathrm{Na}-2 \mathrm{H}[-]$ \\
\hline cotinine methonium ion & NA & DOWN & 1.5 & $5.00 \mathrm{E}-03$ & 209.1516 & 18.8 & $\mathrm{M}+\mathrm{NH} 3+\mathrm{H}[1+]$ \\
\hline trans-3-hydroxycotinine-glucuronide & NA & DOWN & 2.1 & $6.00 \mathrm{E}-03$ & 196.0593 & 12.85 & $\mathrm{M}+\mathrm{H}+\mathrm{Na}[2+]$ \\
\hline \multicolumn{8}{|l|}{ nicotine degradation $I V_{b}$} \\
\hline 3-pyridylacetate & NA & DOWN & 2.1 & 4.50E-03 & 121.0279 & 12.88 & $\mathrm{M}-\mathrm{NH} 3+\mathrm{H}[1+]$ \\
\hline 4-(3-pyridyl)-butanoate & NA & UP & 1.7 & $6.60 \mathrm{E}-03$ & 166.0855 & 13.71 & $\mathrm{M}+\mathrm{H}[1+]$ \\
\hline \multicolumn{8}{|l|}{ sucrose degradationc } \\
\hline$\beta$-D-glucose & 3755 & DOWN & 1.8 & $5.30 \mathrm{E}-03$ & 179.0558 & 11.39 & $\mathrm{M}-\mathrm{H}[-]$ \\
\hline$\beta$-D-glucose & 3755 & DOWN & 3.6 & $1.80 \mathrm{E}-04$ & 181.0704 & 2.79 & $\mathrm{M}+\mathrm{H}[1+]$ \\
\hline$\beta$-D-glucose & 3755 & DOWN & 3.2 & $3.40 \mathrm{E}-04$ & 181.0709 & 2.44 & $\mathrm{M}+\mathrm{H}[1+]$ \\
\hline$\beta$-D-glucose & 3755 & DOWN & 2 & $5.60 \mathrm{E}-03$ & 181.0706 & 11.65 & $\mathrm{M}+\mathrm{H}[1+]$ \\
\hline a-D-glucose & 133 & DOWN & 1.8 & 5.30E-03 & 179.0558 & 11.39 & $\mathrm{M}-\mathrm{H}[-]$ \\
\hline a-D-glucose & 133 & DOWN & 3.6 & $1.80 \mathrm{E}-04$ & 181.0704 & 2.79 & $\mathrm{M}+\mathrm{H}[1+]$ \\
\hline a-D-glucose & 133 & DOWN & 3.2 & $3.40 \mathrm{E}-04$ & 181.0709 & 2.44 & $\mathrm{M}+\mathrm{H}[1+]$ \\
\hline a-D-glucose & 133 & DOWN & 2 & $5.60 \mathrm{E}-03$ & 181.0706 & 11.65 & $\mathrm{M}+\mathrm{H}[1+]$ \\
\hline$\beta$-D-fructofuranose & 63197 & DOWN & 1.8 & 5.30E-03 & 179.0558 & 11.39 & $\mathrm{M}-\mathrm{H}[-]$ \\
\hline$\beta$-D-fructofuranose & 63197 & DOWN & 3.6 & $1.80 \mathrm{E}-04$ & 181.0704 & 2.79 & $\mathrm{M}+\mathrm{H}[1+]$ \\
\hline$\beta$-D-fructofuranose & 63197 & DOWN & 3.2 & $3.40 \mathrm{E}-04$ & 181.0709 & 2.44 & $\mathrm{M}+\mathrm{H}[1+]$ \\
\hline$\beta$-D-fructofuranose & 63197 & DOWN & 2 & $5.60 \mathrm{E}-03$ & 181.0706 & 11.65 & $\mathrm{M}+\mathrm{H}[1+]$ \\
\hline \multicolumn{8}{|l|}{ lactose degradation IIIc } \\
\hline$\beta$-D-galactose & NA & DOWN & 1.8 & $5.30 \mathrm{E}-03$ & 179.0558 & 11.39 & $\mathrm{M}-\mathrm{H}[-]$ \\
\hline$\beta$-D-galactose & NA & DOWN & 3.6 & $1.80 \mathrm{E}-04$ & 181.0704 & 2.79 & $\mathrm{M}+\mathrm{H}[1+]$ \\
\hline$\beta$-D-galactose & NA & DOWN & 3.2 & $3.40 \mathrm{E}-04$ & 181.0709 & 2.44 & $\mathrm{M}+\mathrm{H}[1+]$ \\
\hline$\beta$-D-galactose & NA & DOWN & 2 & $5.60 \mathrm{E}-03$ & 181.0706 & 11.65 & $\mathrm{M}+\mathrm{H}[1+]$ \\
\hline$\beta$-D-glucose & 3755 & DOWN & 1.8 & 5.30E-03 & 179.0558 & 11.39 & $\mathrm{M}-\mathrm{H}[-]$ \\
\hline$\beta$-D-glucose & 3755 & DOWN & 3.6 & $1.80 \mathrm{E}-04$ & 181.0704 & 2.79 & $\mathrm{M}+\mathrm{H}[1+]$ \\
\hline$\beta$-D-glucose & 3755 & DOWN & 3.2 & $3.40 \mathrm{E}-04$ & 181.0709 & 2.44 & $\mathrm{M}+\mathrm{H}[1+]$ \\
\hline$\beta$-D-glucose & 3755 & DOWN & 2 & $5.60 \mathrm{E}-03$ & 181.0706 & 11.65 & $\mathrm{M}+\mathrm{H}[1+]$ \\
\hline \multicolumn{8}{|c|}{ D-galactose degradation V (Leloir pathway)c } \\
\hline$\beta$-D-galactose & NA & DOWN & 1.8 & $5.30 \mathrm{E}-03$ & 179.0558 & 11.39 & $\mathrm{M}-\mathrm{H}[-]$ \\
\hline$\beta$-D-galactose & NA & DOWN & 3.6 & $1.80 \mathrm{E}-04$ & 181.0704 & 2.79 & $\mathrm{M}+\mathrm{H}[1+]$ \\
\hline$\beta$-D-galactose & NA & DOWN & 3.2 & $3.40 \mathrm{E}-04$ & 181.0709 & 2.44 & $\mathrm{M}+\mathrm{H}[1+]$ \\
\hline$\beta$-D-galactose & NA & DOWN & 2 & $5.60 \mathrm{E}-03$ & 181.0706 & 11.65 & $\mathrm{M}+\mathrm{H}[1+]$ \\
\hline a-D-galactose & NA & DOWN & 1.8 & $5.30 \mathrm{E}-03$ & 179.0558 & 11.39 & $\mathrm{M}-\mathrm{H}[-]$ \\
\hline$a-D-g a l a c t o s e$ & NA & DOWN & 3.6 & $1.80 \mathrm{E}-04$ & 181.0704 & 2.79 & $\mathrm{M}+\mathrm{H}[1+]$ \\
\hline a-D-galactose & NA & DOWN & 3.2 & $3.40 \mathrm{E}-04$ & 181.0709 & 2.44 & $\mathrm{M}+\mathrm{H}[1+]$ \\
\hline$a-D$-galactose & NA & DOWN & 2 & $5.60 \mathrm{E}-03$ & 181.0706 & 11.65 & $\mathrm{M}+\mathrm{H}[1+]$ \\
\hline \multicolumn{8}{|l|}{ trehalose degradationc } \\
\hline$\beta$-D-glucose & 3755 & DOWN & 1.8 & $5.30 \mathrm{E}-03$ & 179.0558 & 11.39 & $\mathrm{M}-\mathrm{H}[-]$ \\
\hline$\beta$-D-glucose & 3755 & DOWN & 3.6 & 1.80E-04 & 181.0704 & 2.79 & $\mathrm{M}+\mathrm{H}[1+]$ \\
\hline$\beta$-D-glucose & 3755 & DOWN & 3.2 & $3.40 \mathrm{E}-04$ & 181.0709 & 2.44 & $\mathrm{M}+\mathrm{H}[1+]$ \\
\hline$\beta$-D-glucose & 3755 & DOWN & 2 & 5.60E-03 & 181.0706 & 11.65 & $\mathrm{M}+\mathrm{H}[1+]$ \\
\hline a-D-glucose & 133 & DOWN & 1.8 & $5.30 \mathrm{E}-03$ & 179.0558 & 11.39 & $\mathrm{M}-\mathrm{H}[-]$ \\
\hline a-D-glucose & 133 & DOWN & 3.6 & $1.80 \mathrm{E}-04$ & 181.0704 & 2.79 & $\mathrm{M}+\mathrm{H}[1+]$ \\
\hline a-D-glucose & 133 & DOWN & 3.2 & 3.40E-04 & 181.0709 & 2.44 & $\mathrm{M}+\mathrm{H}[1+]$ \\
\hline a-D-glucose & 133 & DOWN & 2 & $5.60 \mathrm{E}-03$ & 181.0706 & 11.65 & $\mathrm{M}+\mathrm{H}[1+]$ \\
\hline \multicolumn{8}{|l|}{ myo-inositol de novo biosynthesisc } \\
\hline myo-inositol & 144 & DOWN & 1.8 & $5.30 \mathrm{E}-03$ & 179.0558 & 11.39 & $\mathrm{M}-\mathrm{H}[-]$ \\
\hline myo-inositol & 144 & DOWN & 3.6 & $1.80 \mathrm{E}-04$ & 181.0704 & 2.79 & $\mathrm{M}+\mathrm{H}[1+]$ \\
\hline myo-inositol & 144 & DOWN & 3.2 & $3.40 \mathrm{E}-04$ & 181.0709 & 2.44 & $\mathrm{M}+\mathrm{H}[1+]$ \\
\hline myo-inositol & 144 & DOWN & 2 & $5.60 \mathrm{E}-03$ & 181.0706 & 11.65 & $\mathrm{M}+\mathrm{H}[1+]$ \\
\hline \multicolumn{8}{|c|}{ D-myo-inositol $(1,4,5)$-trisphosphate biosynthesisc } \\
\hline myo-inositol & 144 & DOWN & 1.8 & $5.30 \mathrm{E}-03$ & 179.0558 & 11.39 & $\mathrm{M}-\mathrm{H}[-]$ \\
\hline myo-inositol & 144 & DOWN & 3.6 & $1.80 \mathrm{E}-04$ & 181.0704 & 2.79 & $\mathrm{M}+\mathrm{H}[1+]$ \\
\hline myo-inositol & 144 & DOWN & 3.2 & $3.40 \mathrm{E}-04$ & 181.0709 & 2.44 & $\mathrm{M}+\mathrm{H}[1+]$ \\
\hline myo-inositol & 144 & DOWN & 2 & $5.60 \mathrm{E}-03$ & 181.0706 & 11.65 & $\mathrm{M}+\mathrm{H}[1+]$ \\
\hline \multicolumn{8}{|l|}{ glycogenolysisc } \\
\hline$\beta$-D-glucose & 3755 & DOWN & 1.8 & 5.30E-03 & 179.0558 & 11.39 & $\mathrm{M}-\mathrm{H}[-]$ \\
\hline$\beta$-D-glucose & 3755 & DOWN & 3.6 & $1.80 \mathrm{E}-04$ & 181.0704 & 2.79 & $\mathrm{M}+\mathrm{H}[1+]$ \\
\hline$\beta$-D-glucose & 3755 & DOWN & 3.2 & $3.40 \mathrm{E}-04$ & 181.0709 & 2.44 & $\mathrm{M}+\mathrm{H}[1+]$ \\
\hline$\beta$-D-glucose & 3755 & DOWN & 2 & $5.60 \mathrm{E}-03$ & 181.0706 & 11.65 & $\mathrm{M}+\mathrm{H}[1+]$ \\
\hline
\end{tabular}




\begin{tabular}{|c|c|c|c|c|c|c|c|}
\hline Pathway & METLIN ID & Dysregulation* & $\begin{array}{c}\text { Fold } \\
\text { Change }\end{array}$ & p-value & $m / z$ & $t_{R}(\min )$ & Adduct Form \\
\hline \multicolumn{8}{|c|}{ UDP-N-acetyl-D-galactosamine biosynthesis IIc } \\
\hline$\beta$-D-glucose & 3755 & DOWN & 1.8 & $5.30 \mathrm{E}-03$ & 179.0558 & 11.39 & $\mathrm{M}-\mathrm{H}[-]$ \\
\hline$\beta$-D-glucose & 3755 & DOWN & 3.6 & $1.80 \mathrm{E}-04$ & 181.0704 & 2.79 & $\mathrm{M}+\mathrm{H}[1+]$ \\
\hline$\beta$-D-glucose & 3755 & DOWN & 3.2 & $3.40 \mathrm{E}-04$ & 181.0709 & 2.44 & $\mathrm{M}+\mathrm{H}[1+]$ \\
\hline$\beta$-D-glucose & 3755 & DOWN & 2 & $5.60 \mathrm{E}-03$ & 181.0706 & 11.65 & $\mathrm{M}+\mathrm{H}[1+]$ \\
\hline \multicolumn{8}{|l|}{ sorbitol degradation Ic } \\
\hline keto-D-fructose & NA & DOWN & 1.8 & $5.30 \mathrm{E}-03$ & 179.0558 & 11.39 & $\mathrm{M}-\mathrm{H}[-]$ \\
\hline keto-D-fructose & NA & DOWN & 3.6 & $1.80 \mathrm{E}-04$ & 181.0704 & 2.79 & $\mathrm{M}+\mathrm{H}[1+]$ \\
\hline keto-D-fructose & NA & DOWN & 3.2 & $3.40 \mathrm{E}-04$ & 181.0709 & 2.44 & $\mathrm{M}+\mathrm{H}[1+]$ \\
\hline keto-D-fructose & NA & DOWN & 2 & $5.60 \mathrm{E}-03$ & 181.0706 & 11.65 & $\mathrm{M}+\mathrm{H}[1+]$ \\
\hline \multicolumn{8}{|c|}{ lysine degradation I (saccharopine pathway)d } \\
\hline L-saccharopine & 383 & DOWN & 1.9 & $4.90 \mathrm{E}-03$ & 301.1362 & 10.38 & $\mathrm{M}+\mathrm{Na}[1+]$ \\
\hline L-2-aminoadipate & 3271 & DOWN & 2.2 & $5.40 \mathrm{E}-03$ & 185.0652 & 2.47 & $\mathrm{M}+\mathrm{Na}[1+]$ \\
\hline L-2-aminoadipate & 3271 & DOWN & 2.6 & $5.00 \mathrm{E}-03$ & 185.0651 & 2.8 & $\mathrm{M}+\mathrm{Na}[1+]$ \\
\hline L-lysine & 25 & DOWN & 1.9 & $3.70 \mathrm{E}-03$ & 171.1112 & 12.12 & $\mathrm{M}+\mathrm{Na}[1+]$ \\
\hline \multicolumn{8}{|c|}{ lysine degradation II (pipecolate pathway)d } \\
\hline L-2-aminoadipate & 3271 & DOWN & 2.2 & $5.40 \mathrm{E}-03$ & 185.0652 & 2.47 & $\mathrm{M}+\mathrm{Na}[1+]$ \\
\hline L-2-aminoadipate & 3271 & DOWN & 2.6 & $5.00 \mathrm{E}-03$ & 185.0651 & 2.8 & $\mathrm{M}+\mathrm{Na}[1+]$ \\
\hline L-pipecolate & 50 & DOWN & 2 & $2.40 \mathrm{E}-03$ & 150.0546 & 12.8 & $\mathrm{M}+\mathrm{Na}-2 \mathrm{H}[-]$ \\
\hline L-lysine & 25 & DOWN & 1.9 & $3.70 \mathrm{E}-03$ & 171.1112 & 12.12 & $\mathrm{M}+\mathrm{Na}[1+]$ \\
\hline \multicolumn{8}{|l|}{ tRNA charginge } \\
\hline L-histidine & 21 & DOWN & 1.5 & 4.90E-03 & 90.0367 & 2.89 & $\mathrm{M}+\mathrm{H}+\mathrm{Na}[2+]$ \\
\hline L-lysine & 25 & DOWN & 1.9 & $3.70 \mathrm{E}-03$ & 171.1112 & 12.12 & $\mathrm{M}+\mathrm{Na}[1+]$ \\
\hline \multicolumn{8}{|l|}{ histidine degradatione } \\
\hline urocanate & 298 & DOWN & 1.8 & $5.40 \mathrm{E}-03$ & 183.0397 & 9.61 & $\mathrm{M}+\mathrm{HCOO}[-]$ \\
\hline L-histidine & 21 & DOWN & 1.5 & $4.90 \mathrm{E}-03$ & 90.0367 & 2.89 & $\mathrm{M}+\mathrm{H}+\mathrm{Na}[2+]$ \\
\hline \multicolumn{8}{|c|}{ pyrimidine deoxyribonucleotide phosphorylationf } \\
\hline dTTP & 3577 & DOWN & 1.7 & $1.50 \mathrm{E}-04$ & 504.9796 & 10.04 & $\mathrm{M}+\mathrm{Na}[1+]$ \\
\hline \multicolumn{8}{|c|}{$\begin{array}{l}\text { pyrimidine deoxyribonucleotides biosynthesis from } \\
\text { CTPf }\end{array}$} \\
\hline dTTP & 3577 & DOWN & 1.7 & $1.50 \mathrm{E}-04$ & 504.9796 & 10.04 & $\mathrm{M}+\mathrm{Na}[1+]$ \\
\hline \multicolumn{8}{|c|}{$\begin{array}{l}\text { pyrimidine deoxyribonucleotides de novo } \\
\text { biosynthesis } f\end{array}$} \\
\hline dTTP & 3577 & DOWN & 1.7 & $1.50 \mathrm{E}-04$ & 504.9796 & 10.04 & $\mathrm{M}+\mathrm{Na}[1+]$ \\
\hline \multicolumn{8}{|c|}{ dermatan sulfate degradation (metazoa) } \\
\hline a-L-iduronate ${ }^{a}$ & NA & DOWN & 2 & $1.70 \mathrm{E}-03$ & 177.0392 & 13.23 & $\mathrm{M}-\mathrm{H} 2 \mathrm{O}+\mathrm{H}[1+]$ \\
\hline sulfate & 3235 & DOWN & 2.1 & $6.80 \mathrm{E}-03$ & 136.9301 & 1.89 & $\mathrm{M}+\mathrm{K}[1+]$ \\
\hline \multicolumn{8}{|l|}{ oleate biosynthesis } \\
\hline oleate & 190 & DOWN & 3.8 & $6.70 \mathrm{E}-03$ & 153.1264 & 13.58 & $\mathrm{M}+\mathrm{H}+\mathrm{Na}[2+]$ \\
\hline \multicolumn{8}{|l|}{ palmitate biosynthesisg } \\
\hline palmitate & 187 & DOWN & 1.5 & $4.20 \mathrm{E}-03$ & 295.2024 & 19.65 & $\mathrm{M}+\mathrm{K}[1+]$ \\
\hline \multicolumn{8}{|l|}{ stearate biosynthesisg } \\
\hline palmitate & 187 & DOWN & 1.5 & $4.20 \mathrm{E}-03$ & 295.2024 & 19.65 & $\mathrm{M}+\mathrm{K}[1+]$ \\
\hline \multicolumn{8}{|l|}{ a-tocopherol degradation } \\
\hline $\boldsymbol{\alpha}$-carboxyethyhydroxychroman & NA & DOWN & 1.6 & $3.60 \mathrm{E}-05$ & 151.0743 & 18.18 & $\mathrm{M}+\mathrm{H}+\mathrm{Na}[2+]$ \\
\hline \multicolumn{8}{|l|}{ tetrapyrrole biosynthesis } \\
\hline 5-aminolevulinate & 75 & DOWN & 2 & $4.90 \mathrm{E}-03$ & 155.0554 & 9.87 & $\mathrm{M}+\mathrm{Na}[1+]$ \\
\hline \multicolumn{8}{|l|}{ proline degradationh } \\
\hline L-glutamate-5-semialdehyde & 295 & DOWN & 2 & $4.90 \mathrm{E}-03$ & 155.0554 & 9.87 & $\mathrm{M}+\mathrm{Na}[1+]$ \\
\hline proline biosynthesish & & & & & & & \\
\hline L-glutamate-5-semialdehyde & 295 & DOWN & 2 & $4.90 \mathrm{E}-03$ & 155.0554 & 9.87 & $\mathrm{M}+\mathrm{Na}[1+]$ \\
\hline ornithine de novo biosynthesis & & & & & & & \\
\hline L-glutamate-5-semialdehyde & 295 & DOWN & 2 & 4.90E-03 & 155.0554 & 9.87 & $\mathrm{M}+\mathrm{Na}[1+]$ \\
\hline catecholamine biosynthesis & & & & & & & \\
\hline (R)-adrenaline & 62 & UP & 2.3 & $3.10 \mathrm{E}-04$ & 204.0641 & 18.04 & $\mathrm{M}+\mathrm{Na}-2 \mathrm{H}[-]$ \\
\hline 4a-hydroxy-tetrahydrobiopterin & 64413 & UP & 1.7 & $1.90 \mathrm{E}-04$ & 302.1112 & 15.78 & $\mathrm{M}+\mathrm{HCOO}[-]$ \\
\hline L-ascorbatea & 249 & DOWN & 2 & $1.70 \mathrm{E}-03$ & 177.0392 & 13.23 & $\mathrm{M}+\mathrm{H}[1+]$ \\
\hline ascorbate recycling (cytosolic & & & & & & & \\
\hline$\underline{\text { L-ascorbatea }}$ & 249 & DOWN & 2 & $1.70 \mathrm{E}-03$ & 177.0392 & 13.23 & $\mathrm{M}+\mathrm{H}[1+]$ \\
\hline
\end{tabular}

*Dysregulation (fold change) relative to Whites; NA: not applicable

a) Isobaric putative metabolite/metabolites

b) Pathways with overlapping metabolite/metabolites- 1

c) Pathways with overlapping metabolite/metabolites-2

d) Pathways with overlapping metabolite/metabolites- 3

e) Pathways with overlapping metabolite/metabolites-4

f) Pathways with overlapping metabolite/metabolites- 5

g) Pathways with overlapping metabolite/metabolites- 6

h) Pathways with overlapping metabolite/metabolites-7 